\title{
A Compact Squeeze-Film Model Including Inertia, Compressibility, and Rarefaction Effects for Perforated 3-D MEMS Structures
}

\author{
Suhas S. Mohite, Venkata R. Sonti, and Rudra Pratap, Member, IEEE
}

\begin{abstract}
We present a comprehensive analytical model of squeeze-film damping in perforated 3-D microelectromechanical system structures. The model includes effects of compressibility, inertia, and rarefaction in the flow between two parallel plates forming the squeeze region, as well as the flow through perforations. The two flows are coupled through a nontrivial frequencydependent pressure boundary condition at the flow entry in the hole. This intermediate pressure is obtained by solving the fluid flow equations in the two regions using the frequency-dependent fluid velocity as the input velocity for the hole. The governing equations are derived by considering an approximate circular pressure cell around a hole, which is representative of the spatially invariant pressure pattern over the interior of the flow domain. A modified Reynolds equation that includes the unsteady inertial term is derived from the Navier-Stokes equation to model the flow in the circular cell. Rarefaction effects in the flow through the air gap and the hole are accounted for by considering the slip boundary conditions. The analytical solution for the net force on a single cell is obtained by solving the Reynolds equation over the annular region of the air gap and supplementing the resulting force with a term corresponding to the loss through the hole. The solution thus obtained is valid over a range of air gap and perforation geometries, as well as a wide range of operating frequencies. We compare the analytical results with extensive simulations carried out using the full 3-D Navier-Stokes equation solver in a commercial simulation package (ANSYS-CFX). We show that the analytical solution performs very well in tracking the net force and the damping force up to a frequency $f=0.8 f_{n}$ (where $f_{n}$ is the first resonance frequency) with a maximum error within $20 \%$ for thick perforated cells and within $30 \%$ for thin perforated cells. The error increases considerably beyond this frequency. The prediction of the first resonance frequency is within $21 \%$ error for various perforation geometries.

[2007-0001]
\end{abstract}

Index Terms-Gyroscopes, microactuators, microphones, microresonators, microsensors.

\section{INTRODUCTION}

$\mathbf{E}$ FFICIENT compact models for the squeeze-film analysis of perforated microelectromechanical system (MEMS) structures are indispensable in the design of MEMS devices. The most important design goals for dynamic MEMS devices such as accelerometers, microphones, tuning-fork gyroscopes, microswitches, micromirrors, etc., are high sensitivity and high

Manuscript received January 1, 2007; revised February 29, 2008. This work was supported by a grant from the Department of Science and Technology, Government of India. Subject Editor G. Fedder.

The authors are with the CranesSci MEMS Laboratory, Department of Mechanical Engineering, Indian Institute of Science, Bangalore 560012, India.

Color versions of one or more of the figures in this paper are available online at http://ieeexplore.ieee.org.

Digital Object Identifier 10.1109/JMEMS.2008.921675 resolution. When designing MEMS parallel-plate capacitive transducers for these applications, the aforementioned goals can be achieved by maximizing the mechanical compliance $C_{m} \propto\left(A / t^{3}\right)$ and the base capacitance $C_{b} \propto\left(A / h_{a}\right)$ of the structures. This clearly requires sufficiently high surface area $A$ and small thickness $t$ of the vibrating structure, as well as a very small air-gap $h_{a}$ separating the two structures. However, the air in the small gap between the transversely moving planar structure and the fixed substrate imparts damping, spring, and inertial forces to the structures [1]-[3]. These forces have a complex dependence on the air-gap height and the operating frequency. These forces affect the frequency response of the structure and, hence, the sensitivity, resolution, and bandwidth of the device. Damping due to the squeeze-film is considerably high for large surface-to-air-gap ratio. At low frequencies, damping dominates, whereas at high frequencies, spring and inertial effects dominate. Damping can be minimized if it is possible to operate the device under a vacuum condition, which, in turn, requires expensive packaging. However, in cases with high- $Q$ materials, such as silicon, the $Q$-factor of a vibrating system is still mainly determined by the energy losses to the surrounding air as the vacuum in the encapsulated device can hardly be high enough [4]. The amount of squeeze-film damping can be controlled by providing perforations in either the back plate or the oscillating proof mass. These perforations also facilitate the etch release of the sacrificial layer in surface micromachining but reduce the device capacitance, which is undesirable. An adequate value of the base capacitance can be obtained by reducing the air-gap. While reducing the gap, the perforations need to be designed such that constant damping is maintained without sacrificing the capacitance. A feasible range of perforation geometry depends on the chosen micromachining process. To achieve the design goals of minimizing damping and maximizing the capacitance, a tradeoff analysis must be carried out between the perforation geometry and the air-gap. This necessitates creation of simple analytical models and derivation of closed-form formulas for the squeeze-film analysis. These models, in turn, should be validated using finiteelement method (FEM) tools or experimental measurements. The forces due to the squeeze-film extracted from these behavioral models are, in turn, employed in a system-level model for the performance optimization of a MEMS device. Thus, the airgap $h_{a}$ is perhaps the single most crucial parameter that affects the squeeze-film behavior, the base capacitance, and, hence, the overall device performance. However, for small air-gap, the rarefaction and compressibility effects are predominant. In 
addition, at higher frequencies, the compressibility and inertial effects considerably increase and cannot be neglected. In all such applications, where these effects cannot be neglected, the proposed model will be very valuable. Such applications include high-frequency MEMS transducers such as ultrasound transducers (with perforated back plates), moderately high frequency devices with larger air-gap, etc. Although obvious, we add here that the proposed model can, of course, be used in regular cases as well, where compressibility and inertia are negligible.

In the next section, we discuss various phenomena associated with the squeeze-film effect in terms of the nondimensional numbers characterizing them and highlight how they are related to the perforation geometry and the operating frequency of a device.

\section{Modeling RAREFACTION, COMPRESSIBILITY, AND INERTIAL EFFECTS}

\section{A. Rarefaction}

The degree of rarefaction in the fluid flow is characterized by the Knudsen number $K n$. For the air-gap, we define $K n_{g}=$ $\lambda / h_{a}$, where $h_{a}$ is the air-gap thickness, and $\lambda$ is the mean free path of the gas molecules. For the flow through a hole, we define $K n_{h}=\lambda / r_{i}$, where $r_{i}$ is the radius of the hole. The molecular mean free path $\lambda$ is related to the packaging pressure $P$ as $\lambda=P_{a} \lambda_{a} / P$. At ambient conditions, the mean free path $\lambda_{a}$ for air is $0.064 \mu \mathrm{m}$. For MEMS devices with very small airgap and low packaging pressure, the Knudsen number becomes sufficiently high, and the fluid flow transits from a continuum to a rarefied flow. Based on the values of $K n$, the flow regimes are usually divided into four different types: continuum $(K n<$ $0.01)$, rarefaction $(0.01<K n<0.1)$, transition $(0.1<K n<$ $3)$, and molecular $(K n>3)$ regimes [5]. The relative flow rate coefficient $Q_{\mathrm{pr}}(h, p)$ is generally included in the fluid viscosity, and the combined term is called the effective viscosity $\mu_{\mathrm{eff}}=$ $\mu / Q_{\mathrm{pr}}$. Veijola et al. [6] present a simple approximation for the effective viscosity by fitting the respective flow rate coefficients to the experimental values tabulated by Fukui and Kaneko [7]. This relationship is given as $\mu_{\text {eff }}=\mu /\left(1+9.638 K n_{g}^{1.159}\right)$. The value of $\mu_{\text {eff }}$ drops by $30 \%$ (from $\mu$ ) for a $1-\mu \mathrm{m}$ air-gap at 1-atm pressure. For the slip-flow regime, an analytical model is derived using the slip-flow wall boundary conditions on the planar surfaces in the air-gap and on the cylindrical on the hole walls.

\section{B. Compressibility}

The repetitive patterns of pressure distribution observed around the etch holes in a perforated structure can be approximated by equivalent circular pressure cells with a venting boundary condition on the edge of the holes. Consideration of such circular cells considerably simplifies the flow analysis. In the squeeze-film analysis, the extent of compressibility is measured by the squeeze number $\sigma$. An expression for the squeeze number for an annular plate is given in [8] as $\sigma=$ $12 \mu \omega r_{o}^{2} / P_{a} h_{a}^{2}\left((1-\beta)^{2} / \pi^{2}\right)$, where $\beta$ is the ratio of the inner radius to the outer radius of the annulus with a value near unity. A more accurate expression for the squeeze number is derived in [9] by considering the squeeze-film flow, as well as the flow through holes and various other losses. The common parameters in these expressions and in the analytical expressions derived in this paper are given by $12 \mu \omega r_{o}^{2} / P_{a} h_{a}^{2}$. Here, $r_{o}$ is the radius of an equivalent circular pressure cell, which is approximately half the pitch of the holes $\left(r_{o} \approx \xi_{o} / 2\right)$. It is observed that compressibility is proportional to the square of the pitch-to-air-gap ratio $\left(\xi_{o} / h_{a}\right)^{2}$ and the frequency of oscillation $\omega$, and it is inversely proportional to the ambient pressure $P_{a}$. If $\sigma \ll 1$, the compressibility can be neglected, and the flow can be treated as incompressible. For higher values of $\sigma$, the compressibility leads to a significant air-spring effect, which can be undesirable as it can adversely affect the dynamic behavior of a device [10]. Contrary to this, the fact that perforations increase the cutoff frequency-the frequency at which the damping and spring forces are equal-can be exploited by suitably tuning both of these forces by varying the number and size of perforations [11]. Blech [12] and Allen et al. [13] have also reported the use of squeeze-film damping to tailor the frequency response of a seismic accelerometer and that of micromachined sensors, respectively. Therefore, including the compressibility effects in the analysis enables the designer to design the perforation geometry such that the compressibility effects are either totally ruled out or suitably tuned, as the case may be.

\section{Inertia}

The small dimensions of MEMS devices lead to a very small volume, which contains a minuscule quantity of air. Hence, fluid inertia may be neglected at low frequencies $(R e \ll 1)$. However, for larger air-gap heights and at higher frequencies of oscillations, the inertial effects may not be negligible and, hence, need to be considered when calculating the quality factor of such devices [3]. This is done by incorporating the frequency-dependent flow rate coefficient $Q_{\mathrm{pr}}$, which modifies the velocity profile with frequency. For the squeeze-film flow in the air-gap, the modified Reynolds number is defined as $R e_{g}=\rho \omega h_{a}^{2} / \mu$, and for the flow through the hole, it is defined as $R e_{h}=\left(\rho \omega r_{i}^{2}\right) / \mu$. As is evident, the Reynolds number in the two cases increases with the square of the air-gap height and the radius of the hole, respectively.

The analytical model presented in this paper incorporates compressibility, inertia, and gas rarefaction effects and is applicable over a moderate range of perforation geometries and a wide range of frequencies. In the next section, we briefly review various modeling and analysis approaches used for squeezefilm damping of perforated structures.

\section{Modeling Strategies FOR PERForated STRUCTURES}

The squeeze-film flow in dynamic MEMS structures is well modeled by the 2-D Reynolds equation obtained from the Navier-Stokes equation by neglecting inertia and assuming the lateral dimension to be an order of magnitude larger than the air-gap height. For perforated MEMS structures, the 
effective lateral dimension is the pitch of the holes $\xi_{o}$. Therefore, in the case of perforated structures, the assumption of a large lateral dimension begins to deviate. Moreover, the flow through the perforations comes into play, adding an extra dimension to the model. The model dimensionality (2-D or 3-D) is governed by the relative size of the perforation parameters. The significant perforation parameters are the pitch $\xi_{o}$, the diameter $d$, and the length of the holes $l$ (which is the same as the thickness of the perforated plate). Various models have been presented for the squeeze-film analysis of perforated MEMS structures in the literature. These models differ in various respects such as the model dimensionality, the methodologies used, and the different effects considered in the governing equations. Fig. 1 gives an overview of the different perforation geometries and various squeeze-film models at a glance. Solution methods based on numerical, analytical, or mixed techniques are developed to suit the different perforation geometries shown in Fig. 1(a). Numerical methods are best suited for modeling the nonlinear pressure response due to the high amplitudes of oscillation, arbitrary boundary conditions, complex geometries, and nonuniform size and distribution of the holes [10], [15], [16]. Computationally efficient FEM-based simulation schemes are reported in the recent past [17]-[22]. A hybrid model combining the Navier-Stokes equation and the Reynolds equation is proposed in [17]. A mixed-level approach based on FEM and finite network simulation is presented in [18]-[20]. A hierarchical two-level simulation strategy is employed, and a coupled reduced dimensional analysis is performed in [21]. Another method for the arbitrary perforation problem utilizes a Perforation Profile Reynolds solver, which is a multiphysics simulation software [22]. These seminumerical approaches are more efficient than the numerical methods but take time to build the model and require special computational tools.

Our goal of modeling and analyzing the squeeze-film behavior in perforated structures is to perform a reasonably accurate analysis with minimal modeling and computational effort. In this respect, analytical methods are desirable since they give closed-form expressions that can directly be used in systemlevel simulations to evaluate design tradeoffs. In these methods, the repetitive pressure pattern around each hole is exploited, and the entire perforated domain is discretized into pressure cells under simplifying assumptions [9], [23]-[31].

We now discuss different analytical models used for various perforation geometries, as shown in Fig. 1(a.1)-(a.3). When the size of the holes is much larger compared to the air-gap height $\left(d>10 h_{a}\right)$ and the perforations are short $(l / d<1)$, as shown in Fig. 1(a.1), the loss through the holes is not significant. In this case, the problem is reduced to solving the 2-D Reynolds equation under the simplified boundary condition that the acoustic pressure vanishes at the edge of a hole. Closed-form solutions derived assuming incompressible flow exist [23], and solutions including rarefaction, compressibility, and inertial effects are also reported [27]-[29].

For inertial MEMS sensors (e.g., gyroscope and accelerometer), high-aspect-ratio $(l / d>1)$ perforated structures using a deep reactive-ion etching process are designed, as shown in Fig. 1(a.2). In this case, the loss through holes can be significant as the flow is a combination of the horizontal flow between the planar surfaces and the vertical flow through the holes. This problem is modeled by modifying the Reynolds equation by adding a pressure leakage term corresponding to the loss due to the flow through the holes (in the $z$-direction) [24]-[26]. It is assumed that for uniformly distributed holes, the contribution of holes can be homogenized over the entire domain. In these models, the flow is assumed to be incompressible. Alternatively, the flow problem can be treated in two distinct parts. The lateral flow is modeled by the Reynolds equation, and the flow through the holes is modeled by the Poiseuille equation, as done by Homentcovschi and Miles [28]. Their work discusses losses due to flow through holes including inertia but leaves the solution in the squeeze region with a zero-pressure boundary condition. However, in the region just above a hole where the lateral squeeze flow joins the Poiseuille flow, there is a complex interaction causing losses that are difficult to estimate. Moreover, the trivial boundary condition (i.e., $P=0$ ) used in [28] does not hold, and the effect of backpressure due to flow through holes must be accounted for. Darling et al. [30] used arbitrary pressure boundary conditions based on the complex acoustical impedance of an aperture and treated the squeeze-film problem using the Green's function approach. Kwok et al. [31] used the boundary condition on the hole evaluated for incompressible flow in the numerical simulation performed using PDEase, which is a finite-element solver. Under the assumption of incompressible flow (i.e., a low squeeze number), the flow through a hole is merely obtained by geometrical scaling between the cell diameter and the hole diameter. In a recent study, Veijola [9] has used existing analytical models in the squeeze-film and capillary regions, and using FEM simulations, he has derived approximate flow resistances at the intermediate region and at the exit from the hole. The model is valid over a wide range of perforation fractions, from $1 \%$ to $90 \%$. The said study also presents analysis of cutoff frequencies for compressibility and inertia and specifies an upper limit on the frequency up to which the model is valid.

When the pitch of the holes, the air-gap, and the diameter of the holes are all of the same order, as shown in Fig. 1(a.3), the assumptions made in deriving the Reynolds equation do not hold, and the computationally intensive 3-D Navier-Stokes analysis becomes indispensable. Moreover, if the outer borders are open to the ambient, there is a pressure gradient from the center of the plate to its borders, and the pressure patterns around the perforations are not repetitive. In a recent study, a surface extension model has been proposed [32], where the gap size is comparable to the lateral dimensions. In this approach, it is shown that after extending each open border by $(1.3 / 2) h_{a}$, the 2-D Reynolds equation can give accurate results, and the 3-D Navier-Stokes equation can be dispensed with. This approach could be applied to the perforated structures as well.

The central motivation of this paper is to provide closedform expressions to estimate the squeeze-film forces integrated with perforation losses and with more realistic boundary conditions at the holes. When the compressibility and inertial effects, which are prevalent at high operating frequencies, are included in the Reynolds equation, the pressure at the edge of a hole changes with the frequency of oscillations. The venting through the holes acts as a restrictive passage, and the venting 


\begin{tabular}{|c|c|c|}
\hline \multicolumn{3}{|c|}{ (a) Different Perforation Geometries } \\
\hline $1 \longleftarrow \xi_{0} \longrightarrow 1 \quad \nabla^{h_{a}}$ & 1 & $\begin{array}{l}1 \\
1\end{array}$ \\
\hline चाז & $l$ & 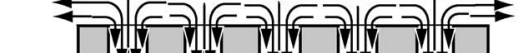 \\
\hline$\frac{\xi_{\mathrm{o}}}{h_{a}} \longrightarrow \quad(>20)$ high & $(10-20)$ moderate & $(<10)$ low \\
\hline$\frac{l}{d} \longrightarrow(<1)$ low & $(>1)$ high & $(\sim 1)$ moderate \\
\hline$\frac{d}{h_{a}} \longrightarrow(>10)$ high & (1-5) moderate & $(\sim 1)$ low \\
\hline Pressure patterns $\longrightarrow$ repetitive & repetitive & non-repetitive \\
\hline $\begin{array}{l}\text { (1) Loss through the holes is negligible: } \\
2 \mathrm{D} \text { squeeze film flow }\end{array}$ & $\begin{array}{l}\text { (2) Loss through the holes is considerable: } \\
\text { 2D squeeze film flow and 1D pipe flow }\end{array}$ & $\begin{array}{l}\text { (3) Small perforation ratios with open } \\
\text { borders leading to nonuniform flow: } \\
\text { Full 3D flow }\left(\xi_{\mathrm{o}} \sim h_{a} \sim l \sim d\right)\end{array}$ \\
\hline
\end{tabular}

\begin{tabular}{|c|c|c|c|c|c|}
\hline \multirow{3}{*}{$\begin{array}{l}\text { (b) Different Modelling Methodologies } \\
\begin{array}{ll}\text { Effects: } & \text { R - Rarefaction, C - Compressibility, I - Inertia } \\
\text { Solution Methods: } & \text { A - Analytical, N- Numerical, } \quad \text { M - Mixed }\end{array}\end{array}$} & \multirow{2}{*}{\multicolumn{3}{|c|}{$\begin{array}{l}\text { Important effects } \\
\text { modelled }\end{array}$}} & \multirow{3}{*}{$\begin{array}{l}\begin{array}{l}\text { Solution } \\
\text { methods }\end{array} \\
\mathrm{A} / \mathrm{N} / \mathrm{M}\end{array}$} & \multirow{3}{*}{$\begin{array}{l}\text { Perforation } \\
\text { geometries } \\
\text { treated }\end{array}$} \\
\hline & & & & & \\
\hline & $R$ & $\mathrm{C}$ & & & \\
\hline $\begin{array}{l}\text { Starr [10]; Reynolds Eq. solved using FEM for large air-gaps assuming incompressible flow and } \\
\text { trivial pressure boundary condition }(P=0) \text {. }\end{array}$ & $x$ & 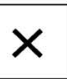 & 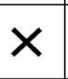 & $\mathrm{N}$ & (a1) \\
\hline Chen et al. [15]; Nonlinear Reynolds Eq. is solved for high amplitudes of oscillations and $(P=0)$. & & X & & $\mathrm{N}$ & 1) \\
\hline Yang et al. [16]; Nonlinear Reynolds Eq. used for flexible structure with large amplitudes and $(P=0)$ & & & & $\mathrm{N}$ & 1) \\
\hline & & & r & M & (a1)-(a3) \\
\hline $\begin{array}{l}\text { Schrag et al. }[18,19], \text { Sattler et al. }[20] ; \text { Mixed level model, accounts for finite size effect and } \\
\text { non-developed flows, uses Finite Network (FN) model of Reynolds Eq., and compact models for edge } \\
\text { effect and perforations, implemented in VHDL-AMS. }\end{array}$ & & & 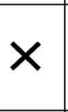 & M & (a1)-(a3) \\
\hline $\begin{array}{l}\text { Råback et al. [21] ; Hierarchical model, first the flow through holes is simulated numerically and } \\
\text { homogenized uniformly on the damper surface in the modified Reynolds equation. }\end{array}$ & & 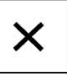 & $<$ & M & (a1)-(a3) \\
\hline $\begin{array}{l}\text { Veijola et al. [22]; Reynolds Eq. modified by adding "leakage" flow through perforations, described } \\
\text { as a spatially variable perforation profile, implemented in Perforation Profile Reynolds (PPR) solver. }\end{array}$ & & & < & M & (a1)-(a3) \\
\hline $\begin{array}{l}\text { Škvor [23]; Expressions for acoustical resistance and inertance are derived for incompressible flow } \\
\text { with trivial venting boundary condition, }(P=0) \text {. }\end{array}$ & $>$ & $<$ & & A & (a1) \\
\hline & & 6 & $<$ & A & (a1)-(a3) \\
\hline $\begin{array}{l}\text { Bao et al. [25, 26]; Reynolds Eq. modified by adding a term for damping effect of gas flow through } \\
\text { holes based on Poiseuille Eq., flow resistance at entry/exit of holes accounted by effective hole length. }\end{array}$ & & X & r & A & (a1)-(a2) \\
\hline $\begin{array}{l}\text { Homentcovschi et al. }[27,28] ; \text { A modified Reynolds Eq. is derived including inertia and solved for } \\
\text { trivial venting boundary condition }(P=0) \text { on holes, similarly equation for losses through holes too are } \\
\text { derived separately, however, the effect of back-pressure due to flow through holes is not incorporated. }\end{array}$ & & & & [27] $\mathrm{A}$ & (a1) \\
\hline Mohite et al. [29]; Compressible Reynolds Eq. solved using trivial venting boundary condition $(P=0)$. & & & $x$ & A & (al) \\
\hline $\begin{array}{l}\text { Darling et al. [30], Reynolds Eq. solved nontrivial venting condition based on the acoustical } \\
\text { impedence of aperture is used. }\end{array}$ & & $x$ & 6 & A & (al) \\
\hline $\begin{array}{l}\text { Kwok et al. [31]; Compressible Reynolds Eq. is used for squeeze-film flow, subsequently, for low } \\
\text { squeeze number }(\sigma) \text { the flow is assumed incompressible and the boundary condition on holes is derived. }\end{array}$ & & $x$ & K & A & (a1)-(a2) \\
\hline $\begin{array}{l}\text { Veijola [9]; Existing analytical models for squeeze-film (Škvor) and capillary regions are augmented by } \\
\text { flow resistances of intermediate region, entry and exit flow, etc., using FEM for incompressible flow. }\end{array}$ & & $x$ & $<$ & $\mathrm{A}$ & (a1)-(a2) \\
\hline $\begin{array}{l}\text { Proposed model; A modified Reynolds equation for squeeze-film flow and Poiseuille equation for flow } \\
\text { through pipe derived including inertia. The flows are coupled through a nontrivial }\left(P=P_{b}\right) \text {, frequency- } \\
\text { dependent pressure boundary condition at the flow junction giving closed-form solutions for 3D flow. }\end{array}$ & & & & $\mathrm{A}$ & $(\mathrm{a} 1)-(\mathrm{a} 2)$ \\
\hline
\end{tabular}

Fig. 1. (a) Different perforation geometries ( $\xi_{o}$ is the pitch of the holes, $h_{a}$ is the air-gap height, and $l$ and $d$ are the length and diameter of the holes, respectively). (b) Overview of different squeeze-film models for perforated structures.

boundary condition becomes a complex acoustical impedance. This boundary condition then depends on the hole geometry (i.e., length and diameter) and flow through the hole. This pressure at the boundary must be evaluated and then applied as a boundary condition at the edge of the hole near the air- gap. This apparently circular computation is circumvented by solving the two flow equations-one for the horizontal flow in the air-gap and the other in the hole-by first assuming an intermediate value of the pressure and then solving for it using two paths of computations discussed in the next section. 


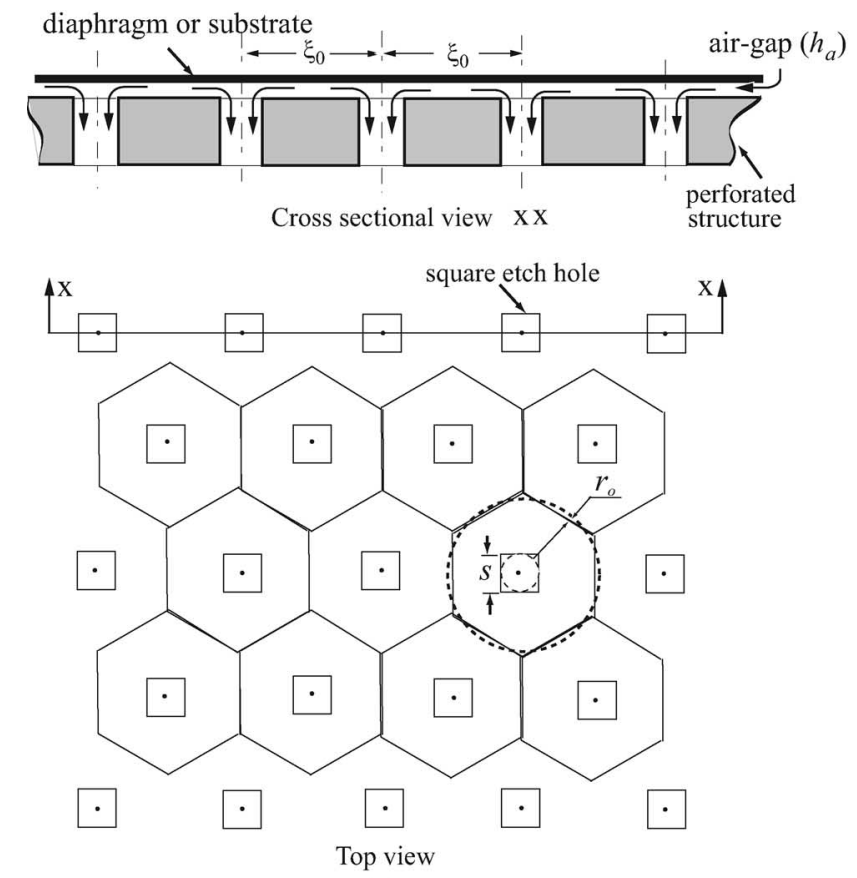

(a)

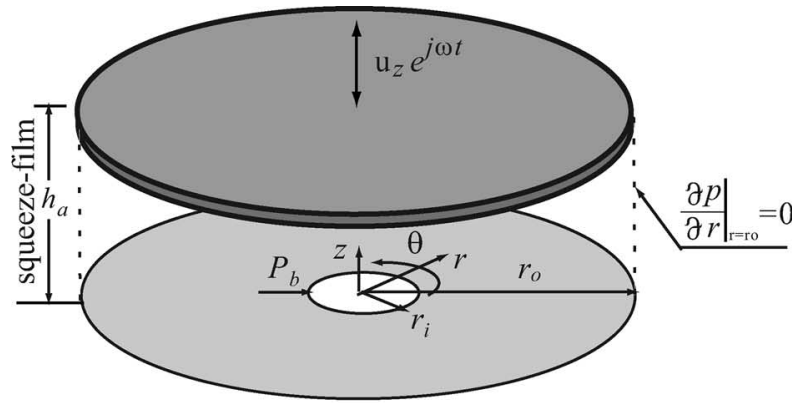

(b)

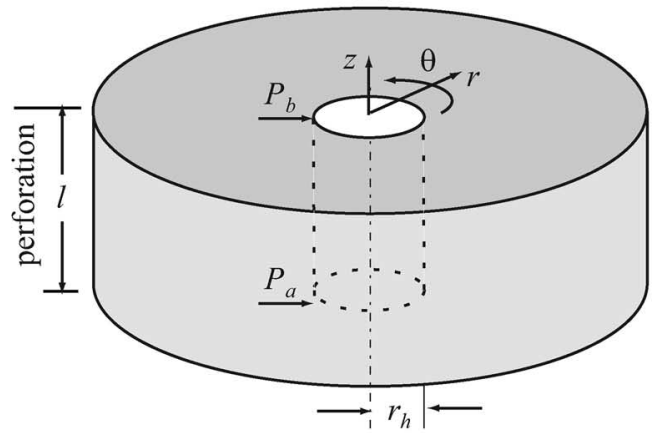

(c)

Fig. 2. Schematic showing the staggered hole configuration. $\xi_{o}$ is the pitch of the holes; $r_{o}=0.525 \xi_{o}$ and $r_{i}=s / \sqrt{\pi}$ are the area equivalent outer and inner radii of the pressure cell, respectively; $r_{h}=s / 2$ is the equivalent hydrodynamic radius of the hole; $h_{a}$ is the air-gap height; and $l$ is the length of the hole (also the thickness of the back plate). (a) Hexagonal pressure patterns in a staggered configuration. (b) Single equivalent circular pressure cell. (c) Poiseuille flow through the perforation.

\section{Analytical Model for a Circular PRESSURE CELL}

\section{A. Modified Reynolds Equation With Inertia, Compressibility, and Gas Rarefaction Effects}

In this section, we present the modified Reynolds equation including unsteady inertia and compressibility, as well as its solution, for a single circular pressure cell under the approximation of 1-D radial flow. Isothermal assumptions are used in deriving the model. The derivation follows the general approach given by Veijola [2], [3] for rectangular plates separated by a small air-gap. We analytically solve the resulting equation, first for a finite-pressure boundary condition at the edge of the hole. Subsequently, in Section IV-B, we show how to compute this pressure at the edge of the hole by analyzing the flow through the hole. A large perforated domain formed by the staggered hole configuration is shown in Fig. 2(a). This domain is discretized into circular pressure cells, and a single pressure cell considered for the analysis is shown in Fig. 2(b). For the squeeze-film problem of a single circular pressure cell, let us assume a constant pressure across the gap $(\partial p / \partial z=0)$ and no net tangential flow $(\partial p / \partial \theta=0)$. The Navier-Stokes momentum equation in cylindrical-polar coordinates then reduces to

$$
\rho \frac{\partial u_{r}}{\partial t}+\rho \frac{\partial u_{r}}{\partial r} u_{r}=-\frac{\partial p}{\partial r}+\frac{\partial}{\partial z}\left(\mu \frac{\partial u_{r}}{\partial z}\right)
$$

where $u_{r}(r, t)$ is the flow velocity in the radial direction, $p(r, t)$ is the air pressure, and $\mu$ and $\rho$ are the viscosity and density of air, respectively. We assume small air-gap compared to the pitch of the holes and small amplitude harmonic oscillations $\left(\epsilon=\epsilon_{o} e^{j \omega t}\right)$. For small air-gap-to-pitch ratios, the lateral velocity $u_{r}$ is relatively small, and the contribution of the convective inertial term $\rho\left(\partial u_{r} / \partial r\right) u_{r}$ can be neglected compared to the unsteady inertial term $\rho\left(\partial u_{r} / \partial t\right)$.

Hence, (1) further reduces to

$$
\rho \frac{\partial u_{r}}{\partial t}=-\frac{\partial p}{\partial r}+\frac{\partial}{\partial z}\left(\mu \frac{\partial u_{r}}{\partial z}\right) .
$$

We nondimensionalize (2) using scaled variables $\Phi=\Delta p / P_{a}$, $Z=z / h_{a}, R=r / r_{o}, \tau=\omega t, U_{z}=\partial \epsilon / \partial \tau$ (i.e., $\left.j \epsilon_{o} e^{j \tau}\right), U_{r}=$ $u_{r} / U_{o}$, and $U_{o}=r_{o} \omega$. Here, $P_{a}$ is the ambient pressure, $h_{a}$ is the equilibrium gap thickness, $\omega$ is the circular frequency, and $r_{o}$ is the outer radius of a pressure cell. The nondimensional form of (2) becomes

$$
\frac{\partial^{2} U_{r}}{\partial Z^{2}}-j R e_{g} U_{r}=\frac{1}{\bar{\sigma}} \frac{\partial \Phi}{\partial R}
$$

where $\bar{\sigma}=\mu \omega r_{o}^{2} / P_{a} h_{a}^{2}$, and the modified Reynolds number $R e_{g}=\rho \omega h_{a}^{2} / \mu$. For very small gaps, or when the pressure is lower than the ambient pressure, the no-slip boundary condition may not hold; therefore, we consider the first-order slip boundary conditions on the two oscillating planar structures as

$$
U_{r}\left(R, \pm \frac{1}{2}\right)=\mp K n_{g} \frac{\partial U_{r}}{\partial Z}\left(R, \pm \frac{1}{2}\right)
$$

where the nondimensional mean free path $K n_{g}=\lambda / h_{a}$ is also the Knudsen number based on the characteristic length $h_{a}$ and the mean free path $\lambda$. When the pressure is lower than the ambient pressure, a quantity $K_{s}=\sigma_{p} K n$ is used as a measure 
of rarefaction, where $\sigma_{p}$ is the slip coefficient [3]. Integrating (3) and applying the boundary conditions (4), we obtain the radial velocity in (5), shown at the bottom of the page.

Integrating (5) w.r.t. $Z$ in the limits $(-1 / 2,+1 / 2)$, we get $\tilde{U}_{r}(R)$ averaged in the $z$-direction as

$$
\tilde{U}_{r}(R)=-\frac{1}{\Gamma} \frac{\partial \Phi}{\partial R}
$$

where $\Gamma=\sigma / Q_{\mathrm{pr}}$ is a nondimensional complex number that includes the compressibility, inertia, and gas rarefaction effects. In the expression for $\Gamma$

$$
\begin{aligned}
Q_{\mathrm{pr}}= & \frac{12}{-j R e_{g}} \\
& \times \frac{\left[\left(2-K n_{g} j R e_{g}\right) \tan \left(\sqrt{-j R e_{g}} / 2\right)-\sqrt{-j R e_{g}}\right]}{\left[\sqrt{-j R e_{g}}\left(1-K n_{g} \sqrt{-j R e_{g}} \tan \left(\sqrt{-j R e_{g}} / 2\right)\right)\right]}
\end{aligned}
$$

is the relative flow rate coefficient that includes the inertial and gas rarefaction effects based on the first-order slip, and $\sigma$ is the nondimensional squeeze number accounting for gas compressibility. The central parameter in $\Gamma$ is the product of the Reynolds number and the squeeze number, i.e., $R e_{g} \sigma$. The significance of this term will be discussed later in Section VI. Next, we derive the modified Reynolds equation in the same manner as the conventional Reynolds equation [3], [14]. For the annular air-gap region, we assume circular symmetry (i.e., $\left.u_{\theta}=0\right)$ and integrate the continuity equation in cylindrical coordinates across the gap from $-h / 2$ to $h / 2$ to get

$$
\frac{1}{r} \frac{\partial\left(\rho r h \tilde{u}_{r}\right)}{\partial r}+\frac{\partial(\rho h)}{\partial t}=0
$$

where $\tilde{u}_{r}$ represents the average velocity such that $\int_{-h / 2}^{+h / 2} u_{r} d z=h \tilde{u}_{r}$.

Furthermore, we nondimensionalize this equation in the same way as (2) and retain only lower order terms in $\epsilon$. Using isothermal flow conditions $(p \propto \rho)$ and substituting $\tilde{U}_{r}$ from (6), we get

$$
\frac{\partial^{2} \Phi}{\partial R^{2}}+\frac{1}{R} \frac{\partial \Phi}{\partial R}-\Gamma\left(\frac{\partial \Phi}{\partial \tau}+\frac{\partial \epsilon}{\partial \tau}\right)=0
$$

Making the transformations $\Psi=\Phi+\epsilon_{o}$ and $\mathcal{R}=\sqrt{j \Gamma} R$ in (8), we get the transformed equation as [33]

$$
\frac{\partial^{2} \Psi(\mathcal{R})}{\partial \mathcal{R}^{2}}+\frac{1}{\mathcal{R}} \frac{\partial \Psi(\mathcal{R})}{\partial \mathcal{R}}-\Psi(\mathcal{R})=0 .
$$

Equation (9) is in the form of the Bessel equation for an imaginary argument of the zeroth order and has a standard solution for a harmonic motion of the diaphragm given as [33]

$$
\Psi=\mathcal{A} I_{0}(\mathcal{R})+\mathcal{B} K_{0}(\mathcal{R})
$$

where $I_{0}$ is the modified Bessel function of the zeroth order, and $K_{0}$ is the Macdonald's function of the zeroth order. $\mathcal{A}$ and $\mathcal{B}$ are complex coefficients that are obtained using the boundary conditions. Substituting back for $\Psi$ and $\mathcal{R}$, we write the normalized complex pressure distribution for the fluid flow governed by (8) over a circular domain for the harmonic velocity of the oscillating plate as

$$
\Phi(R, \tau)=\left\{\mathcal{A} I_{0}(\sqrt{j \Gamma} R)+\mathcal{B} K_{0}(\sqrt{j \Gamma} R)-\epsilon_{o}\right\} e^{j \tau} .
$$

The coefficients $\mathcal{A}$ and $\mathcal{B}$ in (11) are evaluated by substituting the boundary conditions for the pressure cell approximated within a particular pattern. These boundary conditions can be taken as $\left.\Phi\right|_{R_{i}}=0$ and $\partial \Phi /\left.\partial R\right|_{R_{o}}=0$, where $R_{o}$ and $R_{i}$ are the normalized outer and inner radii of the circular pressure cell, respectively. Since the transverse velocity $U_{z}$ of the plate is assumed to be uniform over the pressure cell, the differentiation of (11) yields

$$
\frac{\partial \Phi}{\partial R}(R, \tau)=\sqrt{j \Gamma}\left\{\mathcal{A} I_{1}(\sqrt{j \Gamma} R)-\mathcal{B} K_{1}(\sqrt{j \Gamma} R)\right\} e^{j \tau} .
$$

The zero-radial-gradient boundary condition arises from the assumption of autonomous cells, and the zero-pressure boundary condition at the hole is reasonable for low-aspect-ratio holes. This assumption, however, can be relaxed, and a solution can be obtained in terms of an arbitrary pressure $\Phi_{R_{i}}$ at the hole. In that case, the constants $\mathcal{A}$ and $\mathcal{B}$ are evaluated as

$$
\begin{aligned}
& \mathcal{A}=\frac{\left(\Phi_{R_{i}}+\epsilon_{o}\right) K_{1}(\sqrt{j \Gamma})}{\left[I_{0}\left(\sqrt{j \Gamma} R_{i}\right) K_{1}(\sqrt{j \Gamma})+K_{0}\left(\sqrt{j \Gamma} R_{i}\right) I_{1}(\sqrt{j \Gamma})\right]} \\
& \mathcal{B}=\frac{\left(\Phi_{R_{i}}+\epsilon_{o}\right) I_{1}(\sqrt{j \Gamma})}{\left[I_{0}\left(\sqrt{j \Gamma} R_{i}\right) K_{1}(\sqrt{j \Gamma})+K_{0}\left(\sqrt{j \Gamma} R_{i}\right) I_{1}(\sqrt{j \Gamma})\right]} .
\end{aligned}
$$

Subsequently, the pressure distribution is obtained in (14), shown at the bottom of the page.

Here, $\Phi_{R_{i}}$ is the nondimensional frequency-dependent pressure at the edge of the hole, and its value depends on the backpressure $\Phi_{b}$ at the upper end of the hole. In the next section,

$$
U_{r}(R, Z)=\frac{1}{j R e_{g} \bar{\sigma}}\left(\frac{\cos \left(\sqrt{-j R e_{g}} Z\right)}{\cos \left(\sqrt{-j R e_{g}} / 2\right)-K n_{g} \sqrt{-j R e_{g}} \sin \left(\sqrt{-j R e_{g}} / 2\right)}-1\right) \frac{\partial \Phi}{\partial R}
$$

$$
\Phi(R)=\left[\left(\epsilon_{o}+\Phi_{R_{i}}\right) \frac{I_{0}(\sqrt{j \Gamma} R) K_{1}(\sqrt{\Gamma j})+K_{0}(\sqrt{j \Gamma} R) I_{1}(\sqrt{j \Gamma})}{I_{0}\left(\sqrt{j \Gamma} R_{i}\right) K_{1}(\sqrt{j \Gamma})+K_{0}\left(\sqrt{j \Gamma} R_{i}\right) I_{1}(\sqrt{j \Gamma})}-\epsilon_{o}\right] e^{j \tau}
$$




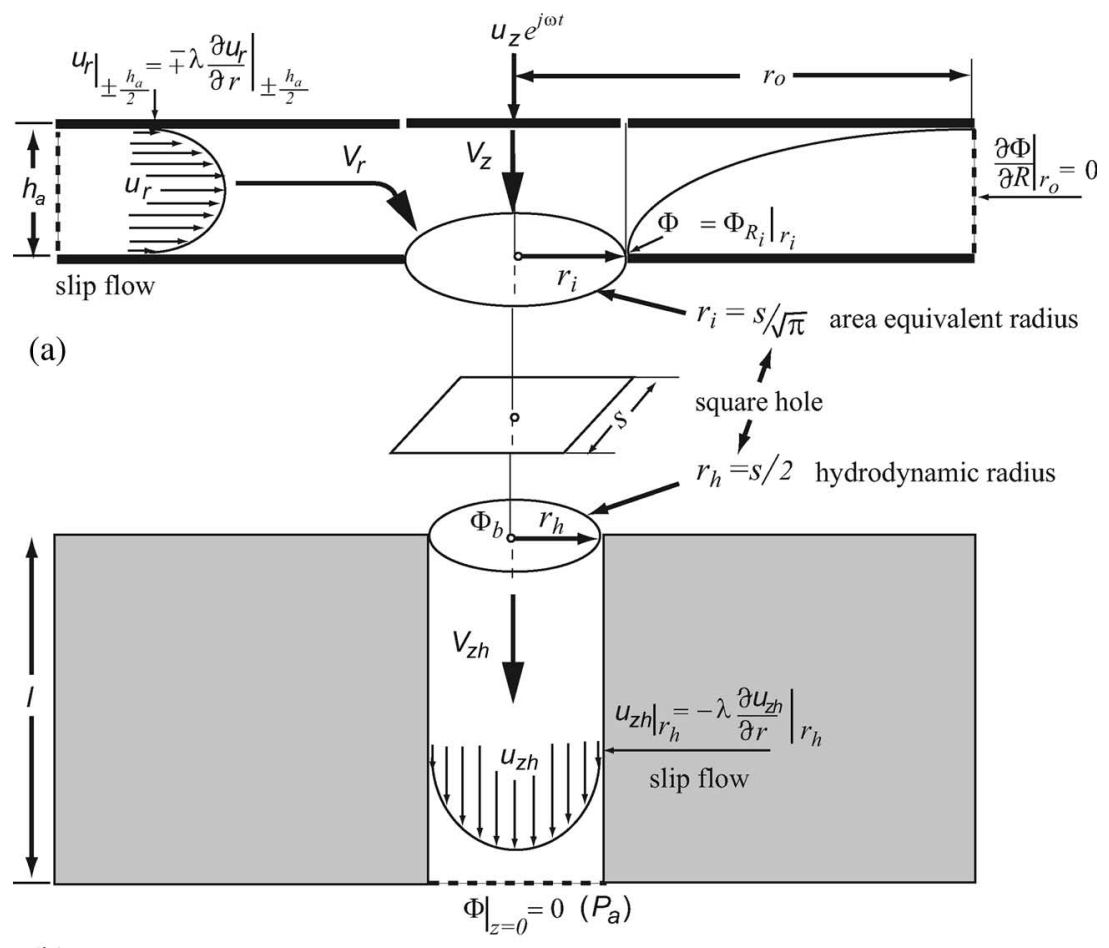

(b)

Fig. 3. Schematic showing the pressure and velocity boundary conditions in different regions. (a) Horizontal squeeze-film flow. (b) Vertical flow through the hole.

we discuss the procedure for obtaining the pressure on the edge of the hole by considering the loss through the hole.

\section{B. Pressure at the Edge of the Hole}

For high-aspect-ratio perforated structures (i.e., $l / d>1$ ), there is a significant loss through the holes that cannot be ignored. In addition, this loss will exert a backpressure on the squeeze-film region, and one cannot apply the trivial pressure boundary condition $(p=0)$ at the edge of the hole. Thus, it becomes necessary to find the pressure at the edge of the hole. Analytical models based on extensive FEM simulations are developed for incompressible flow [31]. However, at high frequencies, the flow velocity in the squeeze-film region given by the modified Reynolds equation incorporating compressibility and inertial effects is frequency dependent. This frequencydependent flow must be used in the Poiseuille equation to obtain a dynamic pressure. Fig. 3 illustrates the problem with the appropriate boundary conditions for the squeeze-film region and for the flow through the hole. We model the motion of the fluid through the hole as a Poiseuille flow through a pipe including inertial effects with atmospheric pressure at the lower end of the hole.

We start by writing the Navier-Stokes equation for the flow through the hole as

$$
\rho \frac{\partial u_{\mathrm{zh}}}{\partial t}=-\frac{\partial p}{\partial z}+\mu \frac{1}{r} \frac{\partial}{\partial r}\left(r \frac{\partial u_{\mathrm{zh}}}{\partial r}\right)
$$

where $u_{\mathrm{zh}}$ is the flow velocity through the hole, and $p$ is the pressure along the axis of the hole. The flow through the holes oscillates with the same frequency $\omega$ as that of the plate. By applying the ambient pressure boundary condition on the free end of the hole, we write (15) as

$$
\frac{1}{r} \frac{\partial}{\partial r}\left(r \frac{\partial u_{\mathrm{zh}}}{\partial r}\right)+\alpha^{2} u_{\mathrm{zh}}=-\frac{p_{b}}{\mu l}
$$

where $p_{b}$ represents the pressure on the upper edge of the hole, and $\alpha=\sqrt{(-j \omega \rho) / \mu}$ is the complex frequency variable $\left(\alpha r_{h}=\sqrt{-j R e_{h}}\right.$, and $R e_{h}=\rho \omega r_{h}^{2} / \mu$ is the modified Reynolds number for the flow through the holes). We solve this equation with $\partial u_{\mathrm{zh}} / \partial r=0$ at $r=0$, and the first-order slip boundary condition $u_{\mathrm{zh}}=-\lambda\left(\partial u_{\mathrm{zh}}\right) / \partial r$ at $r=r_{h}$, to get

$$
u_{\mathrm{zh}}=-\frac{p_{b}}{j \omega \rho l}\left(1-\frac{J_{0}(\alpha r)}{J_{0}\left(\alpha r_{h}\right)-\lambda \alpha J_{1}\left(\alpha r_{h}\right)}\right) .
$$

Veijola [9] has extracted entry and exit losses by performing extensive FEM simulations and presented loss factors in the form of relative elongations. We have not used the relative elongation at the exit as the ambient pressure is applied right at the outlet of the hole in the numerical simulations used for validation. The relative elongation corresponding to the entry loss is used in this analysis, and the effective length of a hole is obtained as $l_{\mathrm{eff}}=\left(l+\Delta c r_{i}\right)$, where $\Delta c$ is given by

$$
\Delta c=\left(1+0.6 K n_{h}\right)\left(0.66-0.41 \frac{r_{i}}{r_{o}}-0.25 \frac{r_{i}^{2}}{r_{o}^{2}}\right) .
$$

By integrating the flow velocity given by (17), we obtain the total volume rate of flow through the hole as 
$V_{\mathrm{zh}}=\int_{0}^{r_{h}} u_{\mathrm{zh}} 2 \pi r d r$. Thus, we get

$$
p_{b}=-\frac{j \omega \rho l_{\mathrm{eff}}}{\left(1-\frac{2}{\alpha r_{h}} \frac{J_{1}\left(\alpha r_{h}\right)}{J_{0}\left(\alpha r_{h}\right)-\lambda \alpha J_{1}\left(\alpha r_{h}\right)}\right)} \frac{V_{\mathrm{zh}}}{\pi r_{h}^{2}} .
$$

We divide $p_{b}$ by the atmospheric pressure $P_{a}$ to get the nondimensional pressure $\Phi_{b}$ on the edge of the hole. Also, we obtain the volume flow rate $\left(V_{\mathrm{zh}}=V_{r}+V_{z}\right)$ through the hole by summing the volume flow rate $\left(V_{r}=2 \pi r_{h} h_{a} \tilde{u}_{r}\right)$ in the radial direction evaluated at $r_{h}$ and the axial volume flow from above the hole $\left(V_{z}=\pi r_{h}^{2} u_{z}\right)$. These substitutions give

$$
\Phi_{b}=\delta_{1}\left(u_{z}+\frac{2 h_{a}}{r_{h}} \tilde{u}_{r}\right)
$$

where

$$
\delta_{1}=\frac{-j \omega \rho l_{\mathrm{eff}}}{P_{a}\left(1-\frac{2}{\alpha r_{h}} \frac{J_{1}\left(\alpha r_{h}\right)}{J_{0}\left(\alpha r_{h}\right)-\lambda \alpha J_{1}\left(\alpha r_{h}\right)}\right)} .
$$

Substituting $\tilde{U}_{r}$ from (6) in $\tilde{u}_{r}=r_{o} \omega \tilde{U}_{r}$, we get

$$
\tilde{u}_{r}=-\frac{r_{o} \omega}{\Gamma} \frac{\partial \Phi}{\partial R}
$$

We first substitute the pressure gradient $\partial \Phi /\left.\partial R\right|_{R=r_{i}}$ given by (14), evaluated at the edge of the hole in (21), to get

$$
\left.\tilde{u}_{r}\right|_{r_{i}}(R)=-\frac{j r_{o} \omega}{\sqrt{j \Gamma}}\left\{\mathcal{A} I_{1}\left(\sqrt{j \Gamma} R_{i}\right)-\mathcal{B} K_{1}\left(\sqrt{j \Gamma} R_{i}\right)\right\} .
$$

Next, we substitute the coefficients $\mathcal{A}$ and $\mathcal{B}$ to get

$$
\left.\tilde{u}_{r}\right|_{r_{i}}=j r_{o} \omega\left(\Phi_{R_{i}}+\epsilon_{o}\right) \delta_{2}
$$

where

$\delta_{2}=-\frac{1}{\sqrt{j \Gamma}}\left[\frac{K_{1}(\sqrt{j \Gamma}) I_{1}\left(\sqrt{j \Gamma} R_{i}\right)-I_{1}(\sqrt{j \Gamma}) K_{1}\left(\sqrt{j \Gamma} R_{i}\right)}{I_{0}\left(\sqrt{j \Gamma} R_{i}\right) K_{1}(\sqrt{j \Gamma})+\left(K_{0} \sqrt{j \Gamma} R_{i}\right) I_{1}(\sqrt{j \Gamma})}\right]$.

Finally, we substitute for $\left.\tilde{u}_{r}\right|_{r_{i}}$ in (20) and obtain the frequency-dependent pressure boundary condition on the edge of the hole as

$$
\Phi_{b}=\delta_{1}\left[u_{z}+\frac{2 h_{a}}{r_{i}} j r_{o} \omega\left(\Phi_{R_{i}}+\epsilon_{o}\right) \delta_{2}\right] .
$$

In (24), we can substitute $\Phi_{R_{i}}=\Phi_{b}$, which turns out to be a reasonable assumption based on the values of pressure gradients in the radial and $z$-directions observed in our simulations. We find that the radial pressure gradient is at least one order of magnitude smaller than the vertical gradient, making the relative pressure variation in the radial direction very small. In fact, the pressure difference between the edge of the hole and the center at the hole opening is between $2 \%$ and $4 \%$ in all simulated cases. Now, solving for $\Phi_{b}$, we get

$$
\Phi_{b}=\frac{\delta_{1}\left(1+\frac{2 r_{o}}{r_{i}} \delta_{2}\right)}{1-j \frac{2 h_{a} r_{o} \omega}{r_{i}} \delta_{1} \delta_{2}} u_{z}
$$

The parameter $\delta_{1}$ depends on the perforation geometry and takes into account the frequency-dependent inertial effects based on the first-order slip flow through the holes. The second parameter $\delta_{2}$ depends on the geometry of the annular squeezefilm region and also takes into account the frequency-dependent compressibility and inertial effects based on the first-order slip flow through the air gap. Substituting $\Phi_{R_{i}}=\Phi_{b}$ in (14), we get the normalized complex force offered by the pressure cell in the annular region as

$$
\mathcal{F}_{\mathrm{sq}}=2 \pi \int_{R_{i}}^{R_{o}} \Phi(R) R d R
$$

Evaluating the above integration over the annular region, we get the complex force due to the squeeze-film $\mathcal{F}_{\text {sq }}=\mathcal{F}_{\text {sq } 1}+$ $\mathcal{F}_{\text {sq2 }}$ given by (27) and (28), shown at the bottom of the page.

Equations (27) and (28) give the squeeze-film force in the annular region of the air-gap due to the small harmonic oscillations of the plate and due to the backpressure caused by the flow through the hole, respectively. Note that for a thin perforated plate and large holes (more venting), the pressure drop through the hole $\Phi_{b}$ is negligible, and the boundary condition approaches the ambient pressure (as one would expect). In that case, the contribution of (28) becomes negligible, and (27) alone can accurately predict squeeze-film damping. Next, the force acting just above the hole is obtained as

$$
\mathcal{F}_{h}=\left[\left(\pi R_{i}^{2}\right) \Phi_{b}\right] e^{j \tau}
$$

The net force on a pressure cell is obtained by adding the forces given by (27)-(29) as

$$
\mathcal{F}_{\text {net }}=\mathcal{F}_{\text {sq } 1}+\mathcal{F}_{\text {sq } 2}+\mathcal{F}_{h}
$$

Finally, we have the damping force and the combined stiffness and inertia force offered by the pressure cell as

$$
\begin{aligned}
\mathcal{F}_{\text {damp. }} & =\left\{\Im\left(\mathcal{F}_{\text {net }}\right)\right\} P_{a} r_{o}^{2} \\
\mathcal{F}_{\text {stiff.+inr. }} & =\left\{\Re\left(\mathcal{F}_{\text {net }}\right)\right\} P_{a} r_{o}^{2}
\end{aligned}
$$

$$
\begin{aligned}
& \mathcal{F}_{\mathrm{sq} 1}=\pi \epsilon_{o}\left[\frac{2 R_{i}}{\sqrt{j \Gamma}} \frac{\left[I_{1}(\sqrt{j \Gamma}) K_{1}\left(\sqrt{j \Gamma} R_{i}\right)-I_{1}\left(\sqrt{j \Gamma} R_{i}\right) K_{1}(\sqrt{j \Gamma})\right]}{\left[I_{0}\left(\sqrt{j \Gamma} R_{i}\right) K_{1}(\sqrt{j \Gamma})+K_{0}\left(\sqrt{j \Gamma} R_{i}\right) I_{1}(\sqrt{j \Gamma})\right]}-\left(1-R_{i}^{2}\right)\right] e^{j \tau} \\
& \mathcal{F}_{\mathrm{sq} 2}=\pi \Phi_{b}\left[\frac{2 R_{i}}{\sqrt{j \Gamma}} \frac{\left.\left[I_{1}(\sqrt{j \Gamma}) K_{1}\left(\sqrt{j \Gamma} R_{i}\right)-I_{1}\left(\sqrt{j \Gamma} R_{i}\right) K_{1}(\sqrt{j \Gamma})\right]\right]}{\left[I_{0}\left(\sqrt{j \Gamma} R_{i}\right) K_{1}(\sqrt{j \Gamma})+K_{0}\left(\sqrt{j \Gamma} R_{i}\right) I_{1}(\sqrt{j \Gamma})\right]}\right] e^{j \tau}
\end{aligned}
$$


TABLE I

Equivalence Between Geometric Variables of ANALYTICAL AND NUMERICAL MODELS

\begin{tabular}{|l|l|}
\hline Description & Parameter \\
\hline Numerical model: & \\
Pitch of the holes & $\xi_{o}$ \\
Length of the side of the square holes & $s$ \\
Length of the square holes & $l$ \\
Analytical model- horizontal squeeze film flow: & \\
(Area equivalent radii of the circular cells [29]) & \\
-For hexagonal patterns in staggered holes & $r_{o}=0.525 \xi_{o}$ \\
-For square patterns in non-staggered holes & $r_{o}=0.564 \xi_{o}$ \\
-Equivalent inner radius of the circular cells & $r_{i}=\frac{s}{\sqrt{\pi}}$ \\
Analytical model- vertical flow through holes: & \\
Equivalent hydrodynamic radius of the holes & $r_{h}=s / 2$ \\
Effective length of the holes [9] & $l_{e f f}=l+\Delta c$ \\
\hline
\end{tabular}

where $\Im$ and $\Re$ represent the imaginary and real parts of their complex arguments, respectively. The phase angle between the imparted velocity and the force response is given as

$$
\theta=\arctan \frac{\Im\left(\mathcal{F}_{\text {net }}\right)}{\Re\left(\mathcal{F}_{\text {net }}\right)} \text {. }
$$

The efficacy of this analytical model is assessed by comparing the analytical results with the numerical simulations using the 3-D Navier-Stokes solver ANSYS-CFX. In the next section, we briefly discuss the numerical model developed in ANSYS-CFX.

\section{Numerical Model AND Simulations}

Generally, perforations are present in staggered and nonstaggered (matrix) configurations. This gives rise to a hexagonal squeeze-film region in the staggered hole configuration and a square squeeze-film region in the nonstaggered hole configuration (in the $x-y$ plane). In addition, square etch hole geometries are very common (in the $z$-direction). In the analytical model, the squeeze-film region and the perforations are assumed to be circular with equivalent radii, as shown in Table I. This simplifies the analytical procedure and gives closed-form solutions. However, in the numerical simulations performed using ANSYS-CFX, the fluid cells are modeled with the actual geometry (i.e., a hexagonal or a square cell with a square hole).

ANSYS-CFX makes use of the finite volume method. Fig. 4 illustrates a typical fluid volume modeled using half-symmetry. The entire fluid domain is discretized using a hexahedral mesh comprising 15000 to 30000 elements for different geometries. A fine mesh is used in the intermediate region above the hole and adjacent to the solid walls. A mesh density of more than 15 elements $/ \mu \mathrm{m}^{3}$ ensures mesh independent analysis, giving a converged solution. The pressure symmetry boundary condition $(\partial p / \partial n=0)$ is applied on the hexagonal periphery.

Losses at the exit of the hole are neglected, and the ambient pressure (i.e., zero relative pressure, $p=0$ ) is applied at the free end of the hole. The flow is assumed to be isothermal, and slip

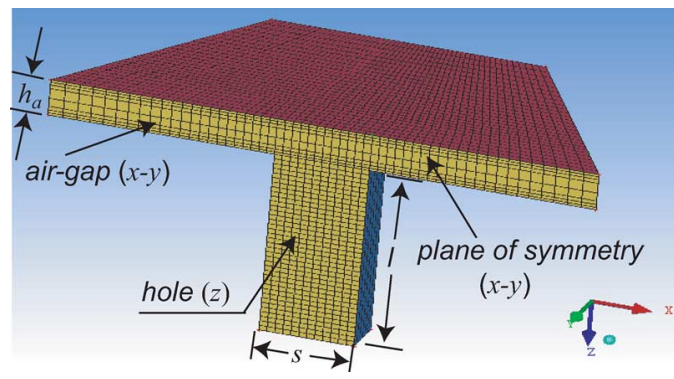

Fig. 4. Hexagonal cell with a square hole (only half-volume) meshed using hexahedral elements for simulations in ANSYS-CFX for $h_{a}=2 \mu \mathrm{m}, s=$ $4 \mu \mathrm{m}$, and $l=10 \mu \mathrm{m}$.

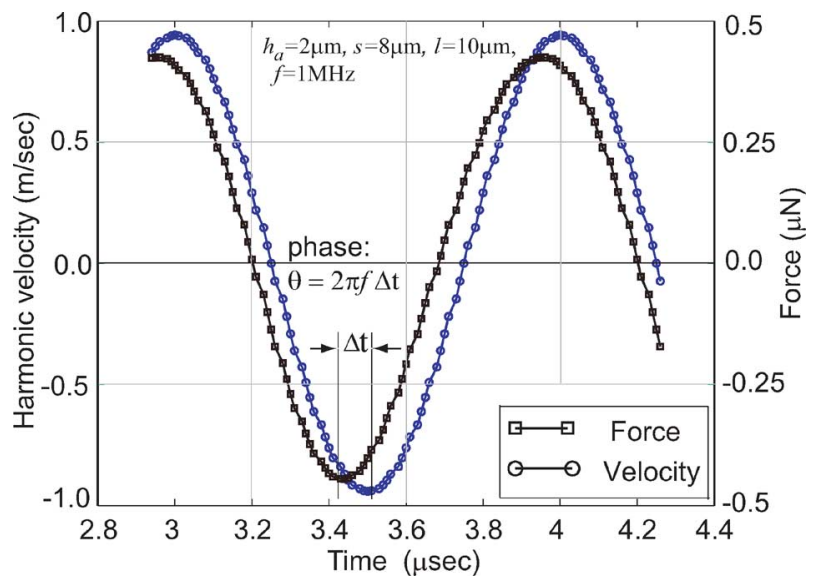

Fig. 5. Steady-state force response to a harmonic velocity applied to the fluid surface in the hexagonal cell simulated in ANSYS-CFX.

boundary conditions are used on the solid walls. A harmonic velocity with a small amplitude of oscillations $\left(\epsilon_{o}=0.075 \times\right.$ $h_{a}$ ), comprising 80 time steps over one cycle of oscillation, is applied normal to the top fluid surface, and transient analysis is performed in each case. We simulated the system response until it reached a steady state. To ensure that the steady state was indeed reached, we computed the response for a few cycles and compared the phase in the current cycle with that in the previous cycle by computing $\left(\theta_{n}-\theta_{n-1}\right) / \theta_{n}$ and restricting this value to less than $1 \%$ to declare convergence. We found that because of the fine time discretization used, convergence was reached within two to ten cycles for all frequencies, with higher frequencies requiring a larger number of cycles. The average pressure acting on the oscillating plate is then extracted, and the net force is obtained by multiplying the average pressure by the area of the plate. Fig. 5 shows a steady-state force response to the imparted harmonic velocity at $1-\mathrm{MHz}$ frequency for a cell with a $2-\mu \mathrm{m}$ air gap and an 8- $\mu \mathrm{m}$-wide $\times 10-\mu$ m-deep perforation. The phase shift between the velocity and the force is calculated as $\theta=2 \pi f \Delta t$, where $\Delta t$ is the time lag or lead between the velocity and the force, and $f$ is the frequency of oscillations. The pressure distributions for the case of hexagonal and square perforated cells are shown in Fig. 6. The primary focus of this study is to examine the behavior of the analytical model that includes compressibility and inertial effects of the fluid and takes into account the loss through the holes. A total of about 500 numerical simulations are carried 

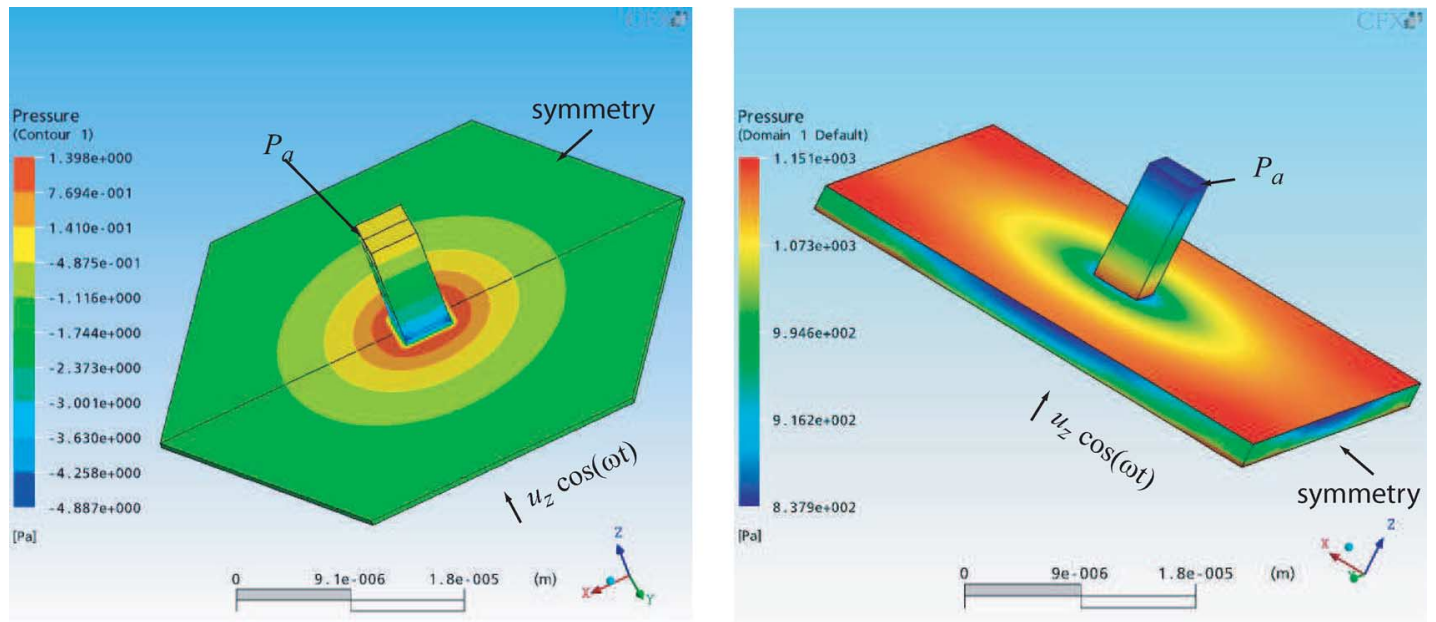

Fig. 6. Pressure profiles in perforated hexagonal and square cells simulated in the 3-D Navier-Stokes solver ANSYS-CFX.

TABLE II

Dimensions and Significant GeOMETRIC Ratios of THE PERforated CElls Modeled fOR Simulations in ANSYS-CFX

\begin{tabular}{|l|l|l|l|}
\hline Description & Parameter & Unit & Value \\
\hline Pitch of the holes & $\xi_{o}$ & $\mu \mathrm{m}$ & 20 \\
Air-gap height & $h_{a}$ & $\mu \mathrm{m}$ & $0.5,1,2,4$ \\
Length of the hole & $l$ & $\mu \mathrm{m}$ & $1,10,20$ \\
Size of the square hole & $s$ & $\mu \mathrm{m}$ & $4,8,10$ \\
Frequency of oscillations & $f$ & $\mathrm{kHz}$ & $5-50,000$ \\
Pitch to air-gap ratios & $\xi_{o} / h_{a}$ & - & $5,10,20,40$ \\
Perforation fractions $(\%)$ & $s^{2} /\left(\pi r_{o}^{2}\right)$ & - & $4.6,18.5,29$ \\
Aspect ratio of the perforations & $l / s$ & - & $0.1-5$ \\
\hline
\end{tabular}

out on 36 different geometries of hexagonal cells. For each geometry, $10-15$ frequency points between $5 \mathrm{kHz}$ and $50 \mathrm{MHz}$ are simulated. The geometries and simulation parameters are summarized in Table II.

The analytical model is based on the frequency-domain form of equations that directly give the frequency response (amplitude of the force), whereas the numerical results are obtained by performing transient analysis at discrete frequencies. In the following sections, we examine the performance of the analytical model by comparing the analytical results with the numerical results over a wide range of frequencies. The results of the comparison are followed by a brief discussion.

\section{RESUlTS AND DISCUSSION}

\section{A. Inertial Effects and Loss Through the Hole}

In our previous work [29], we presented a 2-D analytical squeeze-film damping model incorporating compressibility. In that model, the losses due to the flow through the holes were neglected, and the ambient pressure boundary condition was used on the hole. Moreover, the inertial effects were not modeled. These effects can be significant at high frequencies and for large air-gap heights. Thus, the model was applicable for relatively larger perforations and for low Reynolds number regime

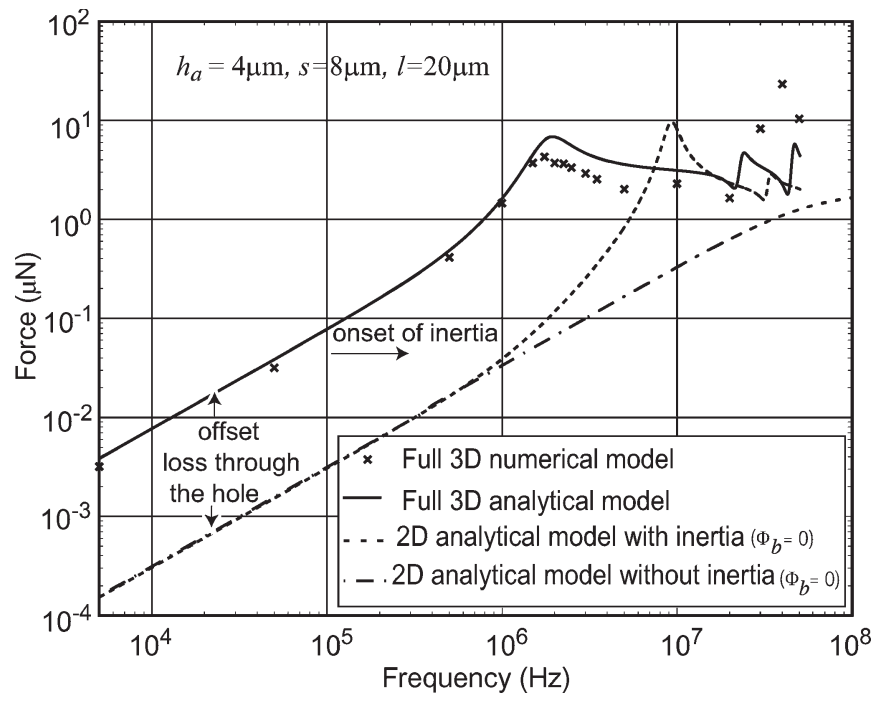

Fig. 7. Frequency response (amplitude) of the squeeze-film model in comparison with the results without inertia and loss through the holes (i.e., $\Phi_{b}=0$ ) and the numerically simulated model in ANSYS-CFX $(\times)$ for $\xi_{o}=20 \mu \mathrm{m}$, $h_{a}=4 \mu \mathrm{m}, s=8 \mu \mathrm{m}$ (perforation ratio of $18.5 \%$ ), and $l=20 \mu \mathrm{m}$.

$(R e<1)$. The motivation for this work is derived from the need to overcome these limitations and develop an improved model. Therefore, we first examine the response of this model, which includes the loss through the hole and inertial effects. For this purpose, we choose a perforation geometry having a $4-\mu \mathrm{m}$ airgap $\left(h_{a}\right)$, an $8-\mu \mathrm{m}$ hole size $(s)$, and a $20-\mu \mathrm{m}$ length of the hole $(l)$. Fig. 7 shows the frequency response results obtained using the analytical model under different assumptions. First, the ambient pressure boundary condition $\Phi_{b}=0$ is set on the upper edge of the hole (i.e., the loss through the hole is neglected), and inertial effects are also suppressed by setting the flow rate coefficient $Q_{\mathrm{pr}}=1$ (i.e., setting $\Gamma=\sigma$ ) in the expression for forces. The frequency response resembling that of a first-order system, consisting of a spring and a damper, shown by a dash-dot line is obtained in this case. Next, the inertial effects in the air-gap are included (i.e., by resetting $\Gamma$ ), but the ambient pressure boundary condition $\Phi_{b}=0$ is still retained. In this case, the inertial effect grows with the frequency of 

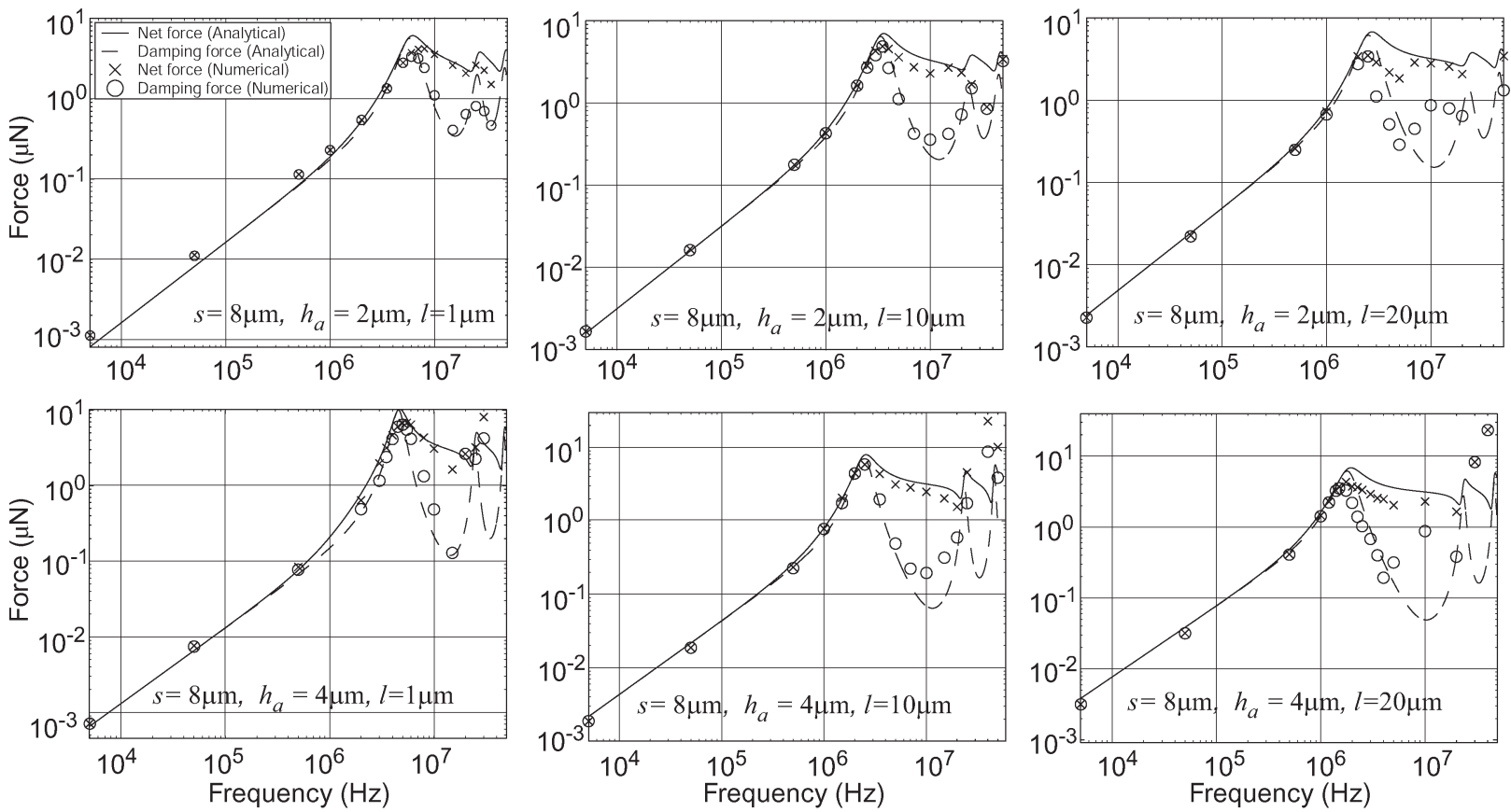

(a)
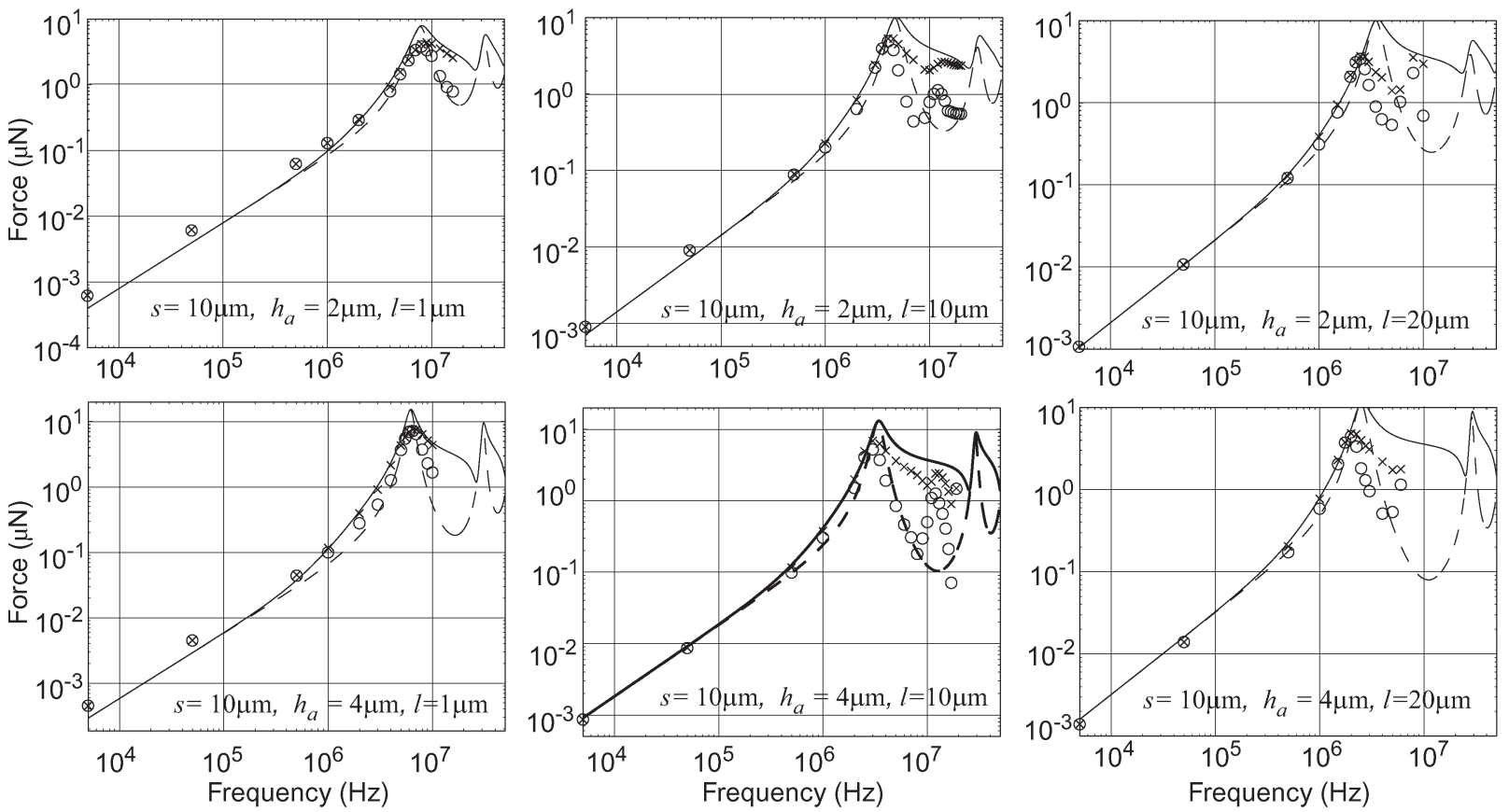

(b)

Fig. 8. Comparison of the net force (solid line) and damping force (dashed line) obtained using analytical formulas with those obtained from ANSYS-CFX ( $\times$ indicates net force, and $\circ$ indicates damping force) for fixed perforation pitch, $\xi_{o}=20 \mu \mathrm{m}$, but varying hole geometry and air gap. (a) Size of the hole $s=8 \mu \mathrm{m}$ (corresponding perforation ratio of 18.5\%), $h_{a}=2$ and $4 \mu \mathrm{m}$, and $l=1,10$, and $20 \mu \mathrm{m}$. (b) Size of the hole $s=10 \mu \mathrm{m}$ (corresponding perforation ratio of $29 \%$ ), $h_{a}=2$ and $4 \mu \mathrm{m}$, and $l=1,10$, and $20 \mu \mathrm{m}$.

oscillations and so does the compressibility effect. The results indicated by a dotted line show a resonance peak, implying that the system behaves as a second-order spring-mass-damper system. Finally, the ambient pressure boundary condition is applied on the lower edge of the hole; thereby, $\Phi_{b}$ gets a finite value corresponding to the loss through the hole, and results of a full 3-D flow are obtained, as shown by the solid line. The results obtained using the numerical model are also plotted for comparison. The offset in the frequency response indicated in
Fig. 7 corresponds to the loss through the hole. The onset of inertial effects is seen from $100 \mathrm{kHz}$ onward, with the solid and dotted lines gradually deviating upward, leading to the first resonance.

\section{B. Comparison of Analytical and Numerical Results}

Next, we compare the behavior of the analytical model with the numerical model for different perforation ratios, air-gap 
TABLE III

Relative Errors in Analytical Resonance Frequencies, Damping Force, and Net Force w.r.t. The Numerical Results for Various GEOMETRIES. THE ERROR Is Estimated OVER Two FREQUENCY REgIMES: L INDICATES THE LINEAR AND MODERATELY NONLINEAR REGION BEFORE THE RESONANCE $\left(f<0.8 f_{n}\right)$, AND NL INDICATES THE STRONGLY NONLINEAR REgion Beyond the Resonance $\left(f>0.8 f_{n}\right)$, ARound the Resonance PEAK

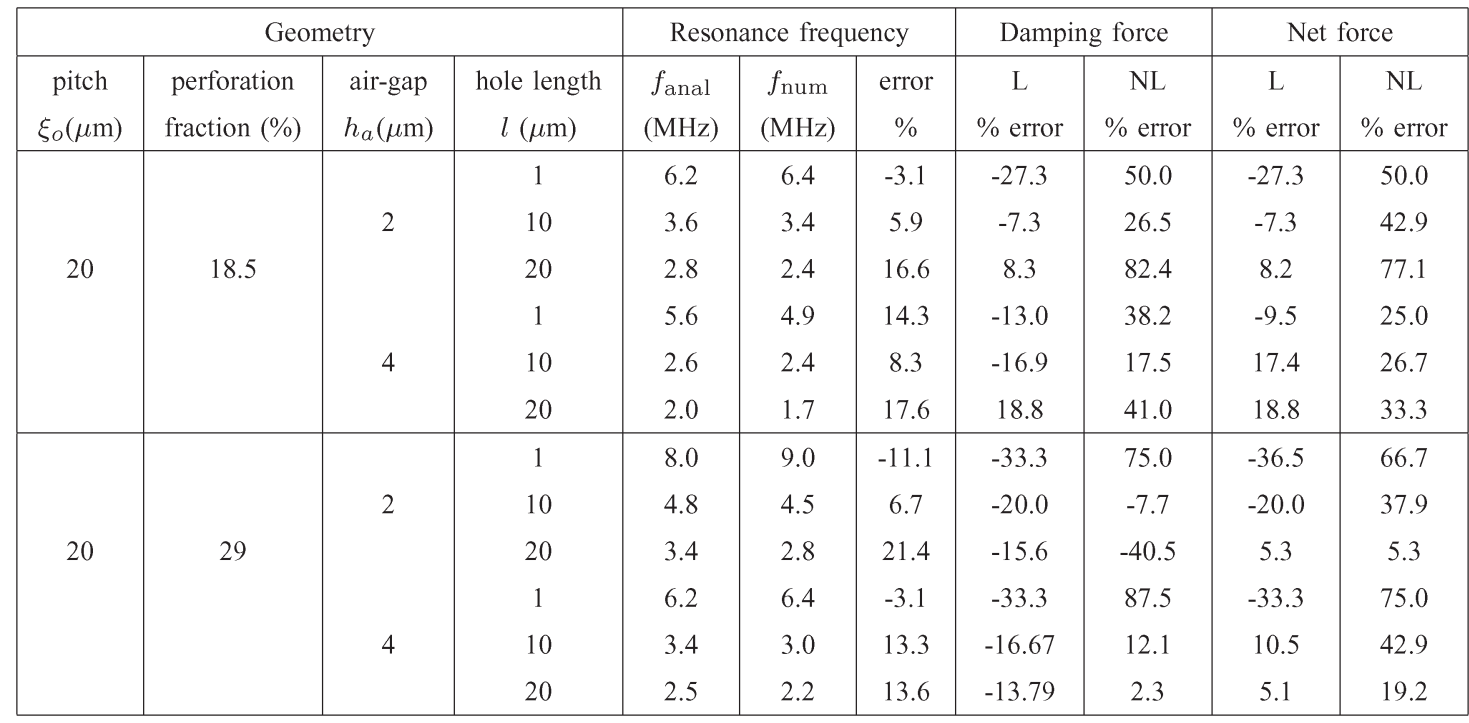

heights, and forcing frequencies. A perforated cell of pitch $\xi_{o}=20 \mu \mathrm{m}$ is selected. Perforation sizes of $s=8 \mu \mathrm{m}$ and $s=10 \mu \mathrm{m}$ give the perforation ratios of $18.5 \%$ and $29 \%$, respectively. These, along with two air-gap heights $h_{a}$ (i.e., 2 and $4 \mu \mathrm{m})$ and three perforation lengths $l$ (i.e., 1,10 , and $20 \mu \mathrm{m}$ ) give 12 combinations. The frequency response curves are shown in Fig. 8(a) and (b). The frequency response amplitude (i.e., the net force) and the damping force due to the squeeze film are very accurately predicted up to a frequency $f=0.8 f_{n}$ (where $f_{n}$ is the first resonance frequency) with less than $20 \%$ error for long hole lengths $(l=10$ and $20 \mu \mathrm{m})$ and less than $30 \%$ error for short hole lengths $(l=1)$. The damping force reaches its peak at the resonance and rapidly decreases beyond the resonance, showing a good agreement with the numerically obtained damping force. However, beyond $0.8 f_{n}$, the frequency response amplitude is overestimated using the analytical model, and in most cases, the error is as high as $80 \%$. This could be due to the fact that the analytical model neglects the convective inertial term. Resonance frequencies predicted by the analytical model and those obtained by interpolating the numerical results are listed in Table III, along with the percentage error. The resonance frequency decreases with the increase in the length of the holes, which is expected (Fig. 8(a) and (b), left to right) due to the increased mass of air in the long holes. Furthermore, when the air-gap increases from 2 to $4 \mu \mathrm{m}$, compressibility drops, and the mass of air increases, lowering the resonance frequencies.

In Fig. 9, we study the effect of perforation size $s$ on the nature of the frequency response. In this case, the perforation length is kept constant at $20 \mu \mathrm{m}$. Four air-gap heights (i.e., 0.5, 1,2 , and $4 \mu \mathrm{m}$ ) and three perforation sizes (i.e., 4,8 , and $10 \mu \mathrm{m}$ ) are considered, giving 12 different geometries. In the case of $4-\mu \mathrm{m}$ perforations, compressibility predominates, and no visible resonance peak is seen for all the air-gap heights. However, in the case of 8 - and $10-\mu \mathrm{m}$ perforation sizes, the resonance peak is seen for all air-gap values except for the $0.5-\mu \mathrm{m}$ airgap. As the frequency of oscillations enters the megahertz regime, the pressure response obtained analytically lies above the response obtained numerically. It should be noted that the nonlinear inertial term $u_{r}\left(\partial u_{r} / \partial r\right)$ is neglected while deriving analytical equations for both the squeeze-film and pipe flows. In particular, beyond the resonance frequency, the contribution of the nonlinear inertial term may not be negligible. The effect is even more pronounced for higher perforation lengths due to the fact that the analytical inertial effect through the pipe is underestimated in the analytical formulas. At even higher frequencies, i.e., beyond $20 \mathrm{MHz}$, the disagreement between the analytical and numerical results is even more, and sometimes, the numerical results drastically fluctuate. At velocities corresponding to these frequencies, one should expect other unmodeled effects such as lateral resonances in the air-gap, etc. The onset of such effects can be detected by a term obtained by the product of the Reynolds number and the squeeze number, i.e., $R e_{g} \sigma=12\left(\left(r_{o} \omega\right)^{2} / c^{2}\right)$ (substituting $P_{a}=\rho c^{2}$, where $c$ is the isothermal sound speed). Since $r_{o} \omega$ is used to normalize the radial velocity $u_{r}$, this ratio is directly useful in predicting the radial flow velocity getting close to acoustically complex phenomena. The term $R e_{g} \sigma$ is independent of the air-gap height and varies with the square of the oscillation frequency. We find that around $20 \mathrm{MHz}$, the comparison between the analytical and numerical models breaks down. At $10-\mathrm{MHz}$ frequency, the ratio $\left(r_{o} \omega\right) / c$ is close to 2 . In actuality, at such high frequencies, the acoustic wavelengths become comparable to the pitch of the pressure cell and the perforation length. The acoustic effects in the lateral direction and in the hole are likely to show up at these frequencies. These effects are not captured by the analytical model. We also point out that at such frequencies, the isothermal flow assumption becomes questionable. At $20 \mathrm{MHz}$, the time period of oscillation is $50 \mathrm{~ns}$, and the time taken for heat propagation from the center of a 

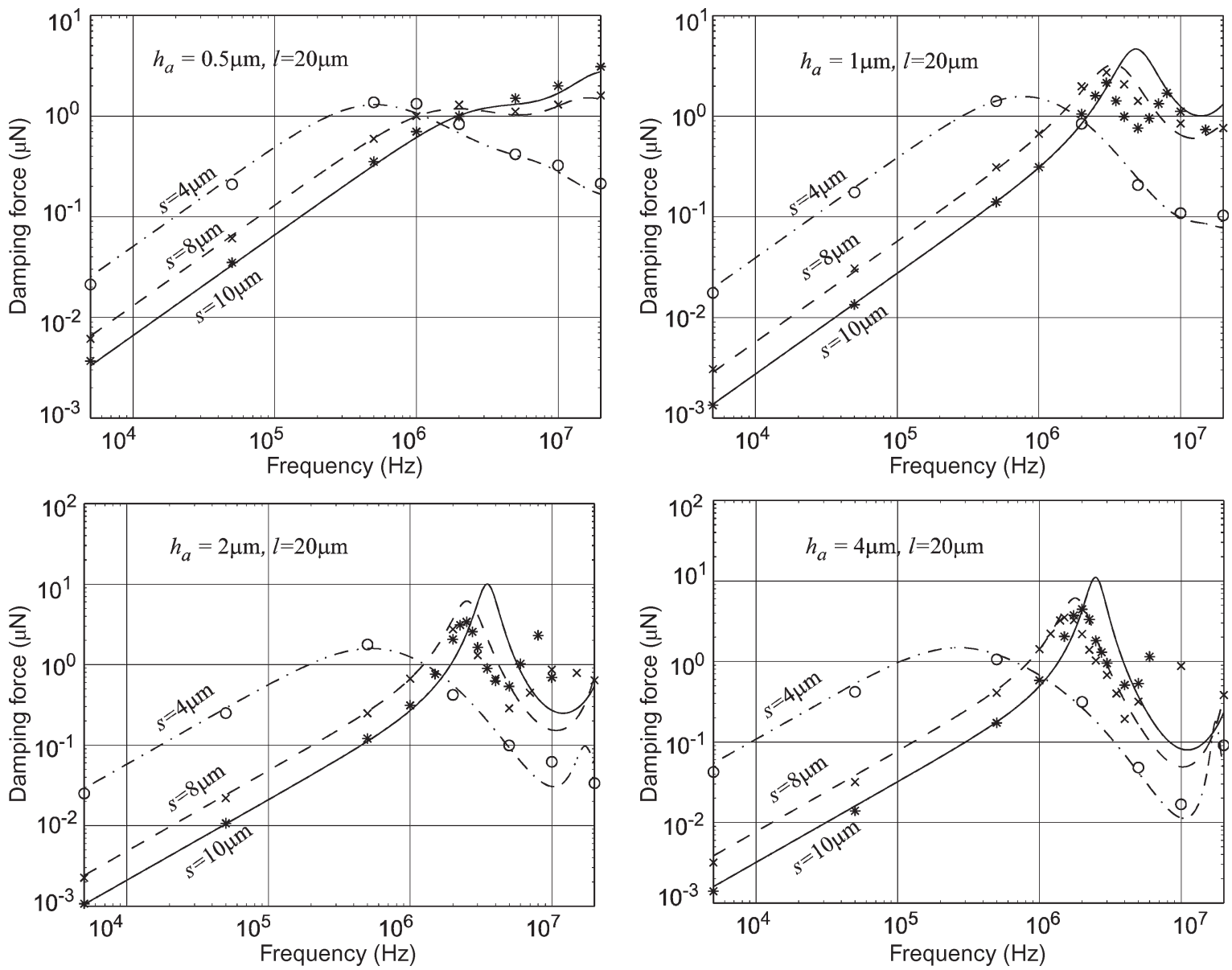

Fig. 9. Comparison of the frequency response curves obtained using analytical formulas and ANSYS-CFX (o, $\times, *$ ) for different air-gap heights and the size of the holes (the pitch of the holes $\xi_{o}=20 \mu \mathrm{m}$, and the length of the holes $l=20 \mu \mathrm{m}$ ).

$2-\mu \mathrm{m}$-thick squeeze film to the solid structural surface (i.e., to travel $1 \mu \mathrm{m}$ ) is about $15 \mathrm{~ns}$ (calculated using the velocity of heat propagation in air, i.e., $v=\sqrt{4 \pi f \kappa}$, where $f$ is the frequency, and $\kappa$ is the diffusivity of air) [34]. Thus, the two timescales are comparable, and the isothermal flow assumption is no longer valid. Therefore, although the analytical results are in good agreement with the numerical results from the ANSYS-CFX simulations until 20-MHz frequency, we recommend using the analytical model only with caution beyond the first resonance peak.

\section{Comparison of Compressibility and Inertial Effects in a 2-D Model}

In Fig. 10, we make a comparison of the frequency response curves obtained using the analytical formulas for four different air-gap heights (i.e., 0.5, 1, 2, and $4 \mu \mathrm{m}$ ). For low frequencies, the response amplitude linearly rises with the frequency of oscillation. This is obviously due to the fact that at low frequencies, the flow is predominantly viscous, which is easily identifiable because both the Reynolds number $R e_{g}$ and the squeeze number $\sigma$ are low $(\ll 1)$. With the increase in frequency, the inertia and compressibility of air in the airgap continuously build up, and for different values of the airgap height, the nature of the response curve considerably varies

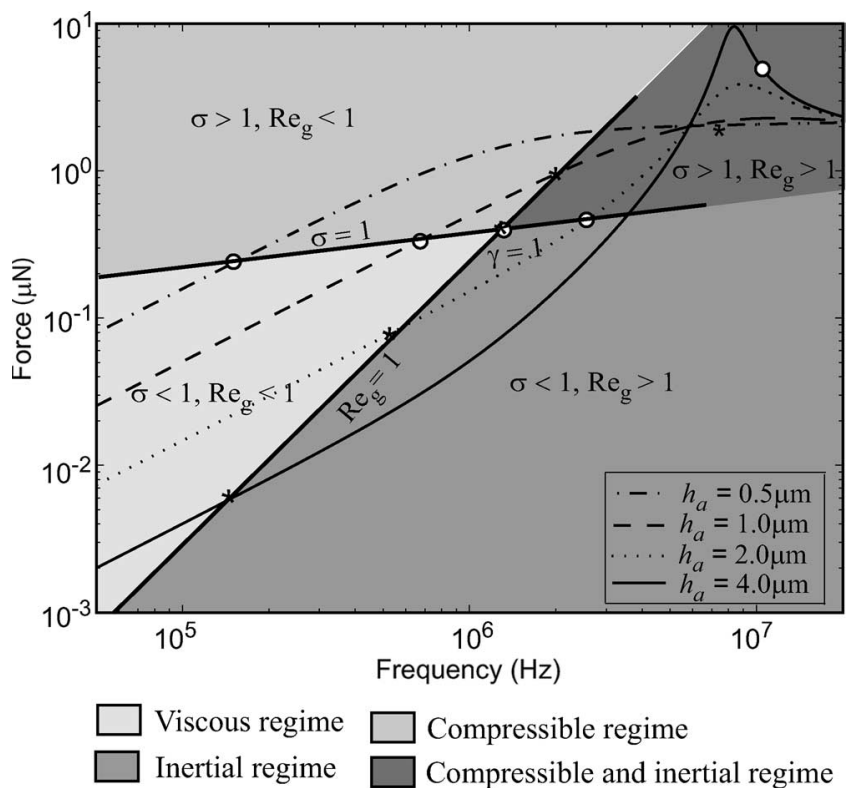

Fig. 10. Comparison of the analytically obtained frequency responses of a 2-D model (i.e., $\Phi_{b}=0$ ) for different air-gap heights (the pitch of the holes $\xi_{o}=20 \mu \mathrm{m}$, and the size of the hole $s=8 \mu \mathrm{m}$ ). Two points are marked on each response curve, where $\sigma=1$ and $R e_{g}=1$. Different shades of gray are used to delineate regions where one or the other effect has a significant effect on force calculations. 
with frequency. The contribution of compressibility and inertia depends on the geometry of the cell and the frequency of oscillations. Since $R e_{g}$ and $\sigma$ linearly vary with frequency, the question is which number rises faster with frequency. This is purely a function of geometry. For a given pitch of the holes and the hole diameter, air-gap $h_{a}$ is the most critical parameter affecting inertia and compressibility. As the air-gap height increases, the inertial contribution, and hence the Reynolds number $\left(\propto h_{a}^{2}\right)$, starts to increase, whereas the compressibility effect $\left(\propto 1 / h_{a}^{2}\right)$ starts to decrease. We propose to use the ratio of the Reynolds number to the squeeze number as a characteristic number to capture the relative effect of inertia and compressibility. Let $\gamma=\left(R e_{g} / \sigma\right)$. For hole size $s=8 \mu \mathrm{m}$ and pitch of the holes $\xi_{o}=20 \mu \mathrm{m}, \gamma=1$ for $h_{a}=1.34 \mu \mathrm{m}$. When $\gamma<1$, compressibility dominates. On the other hand, if $\gamma>1, R e_{g}$ rises faster, which means that inertial effects dominate throughout over compressibility. This is clearly seen from the set of response curves for four different air-gap heights in Fig. 10. For a given geometry, the Reynolds number and the squeeze number are indicative of the frequency at which the inertial and compressibility effects start to dominate over the viscous effects. Since the ratio $\gamma$ eliminates frequency, the effect of geometry is well brought out by this ratio. It is observed that $\gamma$ varies as the fourth power of $h_{a}$, and hence, from $h_{a}=0.5$ to $4 \mu \mathrm{m}$, the ratio goes up by more than three orders of magnitude, switching the behavior from predominantly compressible to mildly compressible and then inertial. This should explain the rapid change from a first-order to a second-order frequency response in Fig. 10. The shaded regions indicate the regimes dominated by the damping, compressibility, and inertial effects.

\section{CONCLUSION}

A compact squeeze-film model was developed for a perforated cell that includes compressibility, inertia, and gas rarefaction effects. The analytical results obtained were compared with the numerical simulations carried out using the finite volume method in ANSYS-CFX. The analytical frequency response and the damping force up to a frequency $f=0.8 f_{n}$ (where $f_{n}$ is the first resonance frequency) match within $20 \%$ accuracy for high-aspect-ratio perforations and 30\% accuracy for lowaspect-ratio perforations. A large error for thinner perforations (smaller hole lengths) suggests that the treatment of orificelike flow with pipe flow (as done here) requires a further refinement to handle this limiting case. The first resonance was predicted within $21 \%$ error depending on the length of the holes. The results clearly show that with the inclusion of inertia and compressibility, the analytical model does very well in predicting the response. However, this model is inadequate for response prediction beyond $f=0.8 f_{n}$ (i.e., around the first resonance frequency) because of the inherent limitations of the fluid flow assumptions made in the derivation of the governing equations. At very high frequencies, the pitch and length of the holes become comparable to the acoustic wavelengths, inviting complex interactions that are not accounted for in this model. Finally, it should be noted that the model presented here is best suited for applications with closed air-gap borders.

\section{ACKNOWLEDGMENT}

The authors would like to thank R. B. Yarasu, CGP Laboratory, for his help in carrying out the Navier-Stokes simulations in ANSYS-CFX and Prof. G. Fedder, Associate Editor, for suggesting a simulation check that led to an improved model for the squeeze-film force calculations. Thanks are also due to A. K. Pandey for valuable inputs.

\section{REFERENCES}

[1] M. Andrews, I. Harris, and G. Turner, "A comparison of squeeze-film theory with measurements on a microstructure," Sens. Actuators A, Phys., vol. 36, no. 1, pp. 79-87, Mar. 1993.

[2] T. Veijola, "Acoustic impedance elements modeling oscillating gas flow in micro channels," in Proc. 4th Int. Conf. Model. Simul. Microsyst., Hilton Head Island, SC, 2001, pp. 96-99.

[3] T. Veijola, "Compact models for squeezed-film dampers with inertial and rarefied gas effects," J. Micromech. Microeng., vol. 14, no. 7, pp. 1109-1118, Jul. 2004.

[4] M. Bao, H. Yang, H. Yin, and Y. Sun, "Energy transfer model for squeezefilm air damping in low vacuum," J. Micromech. Microeng., vol. 12, no. 3, pp. 341-346, May 2002.

[5] G. A. Bird, Molecular Gas Dynamics and the Direct Simulation of Gas Flows. Oxford, U.K.: Oxford Univ. Press, 1996.

[6] T. Veijola, H. Kuisma, J. Lahdenperä, and T. Ryhaanen, "Equivalentcircuit model of the squeezed gas film in a silicon accelerometer," Sens. Actuators A, Phys., vol. 48, no. 3, pp. 236-248, May 1995.

[7] S. Fukui and R. Kaneko, "Analysis of ultra-thin gas film lubrication based on linearized Boltzmann equation: First report-Derivation of a generalized lubrication equation including thermal creep flow," Trans. ASME, J. Tribol., vol. 110, no. 2, pp. 253-262, 1988.

[8] W. S. Griffin, H. H. Richardson, and S. Yamanami, "A study of fluid squeeze-film damping," ASME J. Basic Eng., vol. 88, pp. 451-456, 1966.

[9] T. Veijola, "Analytic damping model for an MEM perforation cell," Microfluid. Nanofluid., vol. 2, no. 3, pp. 249-260, May 2006.

[10] J. B. Starr, "Squeeze-film damping in solid state accelerometers," in Proc. IEEE Solid State Sens. Actuators Workshop, Hilton Head Island, SC, 1990, pp. 44-47.

[11] R. Houlihan and M. Kraft, "Modelling squeeze film effects in a MEMS accelerometer with a levitated proof mass," J. Micromech. Microeng., vol. 15 , no. 5, pp. 893-902, May 2005.

[12] J. J. Blech, "On isothermal squeeze-films," J. Lubr. Technol., vol. 105, pp. 615-620, 1983.

[13] H. V. Allen, S. C. Terry, and D. W. De Bruin, "Accelerometer systems with self-testable features," Sens. Actuators A, Phys., vol. 20, no. 1/2, pp. 153-161, Nov. 1989.

[14] W. A. Gross, Gas Film Lubrication. New York: Wiley, 1962.

[15] C. L. Chen and Y. J. Jason, "Damping control of MEMS devices using structural design approach," in Proc. IEEE Solid State Sens. Actuators Workshop, Hilton Head Island, SC, 1996, pp. 72-75.

[16] Y. J. Yang and S. D. Senturia, "Numerical simulation of compressible squeezed-film damping," in Proc. IEEE Solid State Sens. Actuators Workshop, Hilton Head Island, SC, 1996, pp. 76-79.

[17] M. G. da Silva, "Gas damping and spring effects on MEMS devices with multiple perforations and multiple gaps," in Proc. Transducers, 1999, vol. 2, pp. 1148-1151.

[18] G. Schrag and G. Wachutka, "Physically based modeling of squeeze-film damping by mixed-level system simulation," Sens. Actuators A, Phys., vol. 97/98, pp. 193-200, Apr. 2002.

[19] G. Schrag and G. Wachutka, "Accurate system-level damping model for highly perforated micromechanical devices," Sens. Actuators A, Phys., vol. 111, no. 2/3, pp. 222-228, Mar. 2004.

[20] R. Sattler and G. Wachutka, "Analytical compact models for squeezed-film damping," in Proc. DTIP, Montreux, Switzerland, 2004, pp. 377-382.

[21] P. Råback, A. Pursula, V. Junttila, and T. Veijola, "Hierarchical finite element simulation of perforated plates with arbitrary hole geometry," in Proc. 6th Int. Conf. Model. Simul. Microsyst., San Francisco, CA, 2003, vol. 1, pp. 194-197.

[22] T. Veijola and P. Råback, "A method for solving arbitrary MEMS perforation problems with rare gas effects," in Proc. NSTI-Nanotech, 2005, vol. 3, pp. 561-564.

[23] Z. Škvor, "On the acoustical resistance due to viscous losses in the air gap of electrostatic transducers," Acustica, vol. 19, pp. 295-299, 1967. 
[24] T. Veijola and T. Mattila, "Compact squeezed film damping model for perforated surface," in Proc. Transducers, Munich, Germany, 2001, pp. 1506-1509.

[25] M. Bao, H. Yang, Y. Sun, and P. J. French, “Modified Reynolds' equation and analytical analysis of squeeze-film air damping of perforated structures," J. Micromech. Microeng., vol. 13, no. 6, pp. 795-800, Nov. 2003.

[26] M. Bao, H. Yang, Y. Sun, and Y. Wang, "Squeeze-film air damping of thick hole-plate," Sens. Actuators A, Phys., vol. 108, no. 1-3, pp. 212217, Nov. 2003

[27] D. Homentcovschi and R. N. Miles, "Modeling of viscous damping of perforated planar microstructures. Applications in acoustics," J. Acoust. Soc. Amer, vol. 116, no. 5, pp. 2939-2947, Nov. 2004.

[28] D. Homentcovschi and R. N. Miles, "Viscous damping of perforated planar micromechanical structures," Sens. Actuators A, Phys., vol. 119, no. 2, pp. 544-552, Apr. 2005.

[29] S. S. Mohite, H. Kesari, V. R. Sonti, and R. Pratap, "Analytical solutions for the stiffness and damping coefficients of squeeze films in MEMS devices with perforated back plates," J. Micromech. Microeng., vol. 15, no. 11, pp. 2083-2092, Nov. 2005.

[30] R. B. Darling, C. Hivick, and J. Xu, "Compact analytical modelling of squeeze film damping with arbitrary venting conditions using a Green's function approach," Sens. Actuators A, Phys., vol. 70, no. 1/2, pp. 32-41, Oct. 1998

[31] P. Y. Kwok, M. S. Weinberg, and K. S. Breur, "Fluid effects in vibrating micromachined structures," J. Microelectromech. Syst., vol. 14, no. 4, pp. 770-781, Aug. 2005.

[32] T. Veijola, A. Pursula, and P. Råback, "Extending the validity of squeezed-film damper models with elongations of surface dimensions," J. Micromech. Microeng., vol. 15, no. 9, pp. 1624-1636, Sep. 2004.

[33] N. N. Lebedev, Special Functions and Their Applications. New York: Dover, 1972.

[34] I. B. Crandall, "The air-damped vibrating system: Theoretical calibration of the condenser transmitter," Phys. Rev., vol. 11, no. 6, pp. 449-460, Jun. 1918.

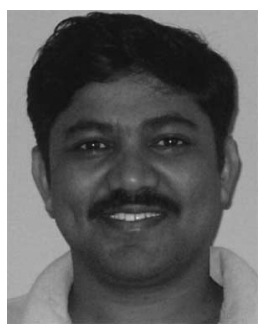

Suhas S. Mohite received the Bachelor's degree in mechanical engineering from the Walchand College of Engineering, Sangli, India, in 1988, the Master's degree in machine tools from the Indian Institute of Technology, Madras, India, in 1990, and the $\mathrm{Ph} . \mathrm{D}$. degree in microelectromechanical systems from the Indian Institute of Science, Bangalore, India, in 2008

He was with Bajaj Auto Ltd., Pune, India, and Wipro Fluid Power Ltd., Bangalore. He is currently a Professor with the Department of Mechanical Engineering, Government College of Engineering, Karad, India. His research interests include dynamics and control, condition monitoring, computeraided design and manufacturing, mechatronics, and design and modeling of MEMS sensors.

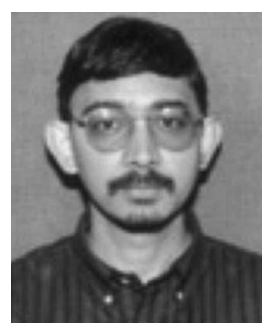

Venkata R. Sonti received the B.Tech. degree from the Indian Institute of Technology, Kharagpur, India, in 1986, and the Ph.D. degree in mechanical engineering from Purdue University, West Lafayette, IN, specializing in digital signal processing, controls, acoustics, and vibration.

Prior to joining the Indian Institute of Science, Bangalore, India, in 1999, he was with Caterpillar, USA, as a Specialist Engineer. He is currently an Assistant Professor with the Department of Mechanical Engineering, Indian Institute of Science. He has worked in active noise control for several years and holds practical skills in product development and testing. His research interests include structural dynamics (vibrations of plates and shells), fluid-structure interaction, acoustics, modeling joints in built-up structures, underwater noise from turbulent flows, development of MEMS acoustic sensors, and sensors for active control of broadband noise.

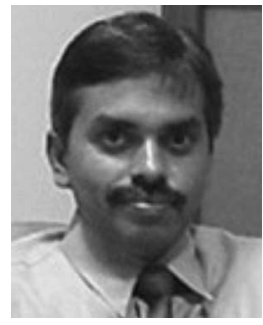

Rudra Pratap (M'07) received the B.Tech. degree from the Indian Institute of Technology (IIT), Kharagpur, India, in 1985, the M.S. degree in mechanics from the University of Arizona, Tucson, in 1987, and the Ph.D. degree in theoretical and applied mechanics from Cornell University, Ithaca, NY, in 1993.

From 1993 to 1996, he was with the Sibley School of Mechanical and Aerospace Engineering, Cornell University. In 1996, he was with the Indian Institute of Science, Bangalore, India. From 1997 to 2000, he was an Adjunct Assistant Professor with Cornell University. He is currently an Associate Professor with the Department of Mechanical Engineering, Indian Institute of Science. His research interests include MEMS design, computational mechanics, nonlinear dynamics, structural vibration, and vibroacoustics.

Dr. Pratap is a member of the Institute of Smart Structures and Systems. He is on the Editorial Board of the journal Computers, Materials, and Continua. 